\title{
Diurnal fluctuations in $\mathrm{CO}_{2}$ and dissolved oxygen concentrations do not provide a refuge from hypoxia and acidification for early-life-stage bivalves
}

\author{
Hannah R. Clark, Christopher J. Gobler* \\ Stony Brook University, School of Marine and Atmospheric Sciences, 239 Montauk Hwy., Southampton, NY 11968, USA
}

ABSTRACT: This study assessed the effects of constant and diurnally fluctuating acidification and hypoxia on the survival, growth, and development of larval stages of 3 bivalves indigenous to the east coast of North America: bay scallops Argopecten irradians, hard clams Mercenaria mercenaria, and eastern oysters Crassostrea virginica. Bivalves were exposed to ideal $(\mathrm{pH}=\sim 7.9$, dissolved oxygen $[\mathrm{DO}]=$ $\left.\sim 7 \mathrm{mg} \mathrm{l}^{-1}\right)$, acidified $\left(\mathrm{pH}=\sim 7.2, \mathrm{DO}=\sim 7 \mathrm{mg} \mathrm{l}^{-1}\right)$, hypoxic $\left(\mathrm{pH}=\sim 7.9, \mathrm{DO}=\sim 2 \mathrm{mg} \mathrm{l}^{-1}\right)$, and acidified and hypoxic $\left(\mathrm{pH}=\sim 7.2, \mathrm{DO}=\sim 2 \mathrm{mg} \mathrm{l}^{-1}\right)$ conditions, as well as treatments that fluctuated between ideal conditions by day and acidified, hypoxic, or acidified and hypoxic conditions by night. Continuously acidified conditions reduced survival of larvae of all 3 species, slowed growth of larval bay scallops and eastern oysters, and delayed the development of bay scallop larvae. Continuously hypoxic conditions reduced the survival, growth, and development of larval bay scallops and slowed the development of larval hard clams. Simultaneous exposure to continuously low $\mathrm{pH}$ and DO yielded more negative effects than each factor independently. Diurnal exposure to low $\mathrm{pH}$ and/or low DO rarely altered, and never fully mitigated, the negative effects of hypoxia and/or acidification despite significantly higher mean $\mathrm{pH}$ and DO levels. This suggests that $\mathrm{pH}$ and DO fluctuations were too intense, and/or the durations of normoxic and normcapnic conditions were not long enough for bivalve larvae to overcome the physiological stress of hypoxia and acidification. Therefore, the diurnal fluctuations of $\mathrm{pH}$ and $\mathrm{DO}$ in this study did not provide a temporal refuge from hypoxia and acidification for North Atlantic bivalve larvae, suggesting that such fluctuations in an ecosystem setting can be a significant threat to these larvae.

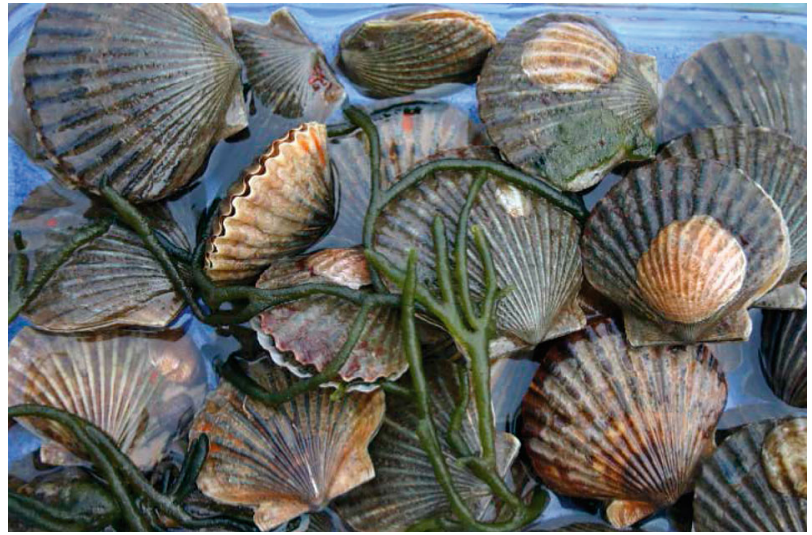

Periods of higher DO and $\mathrm{pH}$ within diurnal fluctuations do not provide a temporal refuge from hypoxia and acidification for larvae of bivalves such as the adult bay scallop Argopecten irradians shown here. Such fluctuations can represent a significant environmental threat at the ecosystem level.

Photo: Stephanie Talmage-Forsberg

KEY WORDS: Ocean acidification $\cdot$ Hypoxia $\cdot$ Estuary · Bivalve $\cdot$ Larvae $\cdot$ Ecosystem metabolism $\cdot$ Diurnal patterns

\section{INTRODUCTION}

During the past 2 centuries, the ocean has assimilated nearly $30 \%$ of anthropogenic $\mathrm{CO}_{2}$ emissions, leading to declining levels of $\mathrm{pH}$, carbonate $\left(\mathrm{CO}_{3}{ }^{2-}\right)$ ions, and saturation state of calcium carbonate (Sabine et al. 2004, Caldeira \& Wickett 2003). Coastal ecosystems are also susceptible to excessive $\mathrm{CO}_{2}$ from eutrophication (Wallace et al. 2014). Algal growth stimulated by excessive nutrients delivers organic matter to

( $)$ The authors 2016. Open Access under Creative Commons by Attribution Licence. Use, distribution and reproduction are unrestricted. Authors and original publication must be credited. 
bottom waters, where microbial respiration driven by the degradation of this organic matter can deplete oxygen, produce $\mathrm{CO}_{2}$, and acidify the water (Cai et al. 2011, Melzner et al. 2013, Wallace et al. 2014). While hypoxia in coastal zones has been studied for decades, the concurrent acidification of hypoxic zones has only recently been well-documented (Cai et al. 2011, Melzner et al. 2013, Baumann et al. 2015).

Although there is consensus that hypoxia in coastal systems is exacerbated by anthropogenic nutrient loading (Diaz 2001, Rabalais et al. 2002, Breitburg et al. 2009), there is some debate regarding how atmospherically driven ocean acidification will manifest in coastal regions. Unlike the open ocean, where pH remains relatively constant, coastal systems are typically less buffered (Cai \& Wang 1998, Wang et al. 2013) and biological activity, combined with other variables, can drive $\mathrm{pH}$ fluctuations on diurnal and/or seasonal timescales (Ringwood \& Keppler 2002, Wootton et al. 2008, Waldbusser \& Salisbury 2014). During the day, photosynthetic activity produces oxygen, consumes $\mathrm{CO}_{2}$, and increases $\mathrm{pH}$, whereas at night, respiration becomes the dominant metabolic reaction consuming oxygen, producing $\mathrm{CO}_{2}$, and decreasing $\mathrm{pH}$ (Yates et al. 2007, Wootton et al. 2008). Some have hypothesized that this metabolic control on $\mathrm{pH}$ is so great that the effects of ocean acidification will be small relative to the fluctuating diurnal acidification in estuaries and coastal waters (Duarte et al. 2013), or that metabolic activity driven by eutrophication may overwhelm the effects of ocean acidification in coastal surface waters (Borges \& Gypens 2010). Alternatively, it has been suggested that ocean acidification will affect the magnitude of metabolically driven fluctuations in $\mathrm{pH}$ by decreasing the baseline $\mathrm{pH}$ of coastal systems (Miller et al. 2009, Feely et al. 2010). Coastal systems, being less buffered (Cai \& Wang 1998, Wang et al. 2013), may then experience changes from ocean acidification before changes are observed in the open ocean (Waldbusser et al. 2011). In addition, Sunda \& Cai (2012) predicted that future $\mathrm{CO}_{2}$ concentrations in eutrophic coastal systems will increase nonlinearly from the combined delivery of $\mathrm{CO}_{2}$ from the atmosphere and respiration. Cai et al. (2011) compared models of the pre-industrial and present-day conditions in the Gulf of Mexico and reported a decrease in $\mathrm{pH}$ of 0.45 units, noting that acidified ocean waters and eutrophicationdriven respiration contributed to the decrease by 0.11 and $0.29 \mathrm{pH}$ units, respectively. Records of $\mathrm{pH}$ in the waters surrounding Tatoosh Island, WA, USA, showed a significant decrease from 2000 to 2007 despite the presence of large diurnal fluctuations as a result of metabolism (Wootton et al. 2008).
Hypoxia increases stress and mortality in shellfish and other organisms (Diaz \& Rosenberg 1995, Vaquer-Sunyer \& Duarte 2008, Breitburg et al. 2009). Tolerance to hypoxia, however, can vary among shellfish species and age, and can interact with other stressors (Wu 2002, Zhang et al. 2010). Earlier life stages of bivalves are generally more vulnerable to low oxygen (Wang \& Widdows 1991, 1993, Gobler et al. 2014). In addition, some bivalves undergo anaerobic respiration as dissolved oxygen (DO) concentrations decline, an adaptation that may permit greater resistance to the effects of low DO (Wu 2002). Importantly, however, the potential importance of anaerobiosis for bivalves increases with age and can only comprise $10 \%$ of total metabolism within post-larval individuals (Wang \& Widdows 1991, 1993), a fact that may further account for the higher vulnerability of early life stages to hypoxia and acidification (Gobler et al. 2014).

Acidification can be inhibitory to calcifying bivalves, potentially inducing mortality, delayed metamorphosis, and slowed growth and calcification rates in early life stages (Talmage \& Gobler 2009, 2010, Gazeau et al. 2013, Waldbusser et al. 2013, White et al. 2013). Less is known regarding the effects of the simultaneous exposure of low DO and low $\mathrm{pH}$ on shellfish. Some studies have found that low oxygen effects tend to dominate over $\mathrm{pH}$ effects (Kim et al. 2013), while others have found that the combined effects of acidification and hypoxia are more severe than the effects of each individual stressor, and that impacts in bivalves can be age-dependent (Gobler et al. 2014, Steckbauer et al. 2015). Keppel et al. (2015) examined how diurnal cycling of DO and pH affected disease acquisition in eastern oysters Crassostrea virginica, and found that diurnal cycling of DO increased the acquisition and progression of Perkinsus marinus infections during exposure and had a legacy effect the next year.

The goal of this study was to quantify the effects of static and diurnally fluctuating low DO and low pH on larval bay scallops Argopecten irradians, hard clams Mercenaria mercenaria, and eastern oysters $C$. virginica native to North Atlantic estuaries. While the effects of hypoxia or acidification on early-life-stage bivalves are well-known, their combined effects have been poorly studied, despite their frequent cooccurrence in estuaries (Cai et al. 2011, Melzner et al. 2013, Wallace et al. 2014). As coastal systems often experience metabolically driven, diurnal cycles of $\mathrm{pH}$ and DO, this study investigated how early-life-stage bivalves responded to diurnal patterns of acidification and hypoxia. Although high partial pressure of $\mathrm{CO}_{2}\left(p \mathrm{CO}_{2}\right)$ and low DO concentrations are known to 
negatively affect larval bivalves, it was hypothesized that the negative effects would be lessened or mitigated when exposure to such conditions was in the form of repeated, short-term diurnal cycles. Physiological adaptations of shellfish, such as acid-base regulation and metabolic depression (Wu 2002, Michaelidis et al. 2005), may allow these bivalves to tolerate excursions into hypercapnic and hypoxic conditions, and once favorable conditions return, compensatory growth may occur and development may continue uninhibited.

\section{MATERIALS AND METHODS}

\section{Manipulation of pH and DO}

Replicate $(n=4) 81$ polyethylene vessels were used for experiments and filled with UV-sterilized, $0.2 \mu \mathrm{m}$ filtered seawater from Old Fort Pond in Shinnecock Bay, NY, USA (salinity $=30$ ). A constant temperature of 23 to $24^{\circ} \mathrm{C}$ was maintained by partially submerging the experimental vessels in a water bath heated by a Delta Star heat pump. Experiments manipulating $\mathrm{pH}$ and DO were performed with each species of bivalve larvae, whereby control $\left(\mathrm{pH}=\sim 7.9\right.$, DO $\left.=\sim 7.0 \mathrm{mg} \mathrm{l}^{-1}\right)$, low $\mathrm{pH}$ and normal DO $\left(\sim 7.2, \sim 7.0 \mathrm{mg} \mathrm{l}^{-1}\right.$, respectively), normal $\mathrm{pH}$ and low DO $\left(\sim 7.9, \sim 2.0 \mathrm{mg} \mathrm{l}^{-1}\right.$, respectively), and combined low $\mathrm{pH}$ and $\mathrm{DO}(\sim 7.2, \sim 2.0 \mathrm{mg}$ $\mathrm{l}^{-1}$, respectively) treatments were maintained by bubbling mixtures of air and tanked $5 \times 10^{4} \mathrm{ppm} \mathrm{CO}_{2}$, pure $\mathrm{N}_{2}$, and a $400 \mathrm{ppm} \mathrm{CO}_{2}+\mathrm{N}_{2}$ mix into experimental vessels (Gobler et al. 2014). Additional treatments of diurnally fluctuating $\mathrm{pH}$, diurnally fluctuating $\mathrm{DO}$, and combined diurnally fluctuating $\mathrm{pH}$ and $\mathrm{DO}$ were also carried out, where $\mathrm{pH}, \mathrm{DO}$, or both parameters oscillated between control and low conditions every $12 \mathrm{~h}$ (details below; Fig. 1), creating a mean pH and DO exposure level higher than the continuously low DO and/or $\mathrm{pH}$ exposures. Both the absolute levels of $\mathrm{pH}$ and $\mathrm{DO}$, and the diurnal patterns of $\mathrm{pH}$ and DO applied in experiments, were consistent with prior observations in eutrophic estuaries (Ringwood \& Keppler 2002, Melzner et al. 2013, Wallace et al. 2014, Baumann et al. 2015). Further, the level of DO and $\mathrm{pH}$ selected were previously found to be harmful to bivalve larvae individually (Wang \& Widdows 1991, 1993, Vaquer-Sunyer \& Duarte 2008, Talmage \& Gobler 2010), but have yet to be explored in unison under static or fluctuating conditions.
The delivery rate of gases was controlled with a series of Cole-Parmer gas regulators, single-tube flowmeters, and/or multi-tube gas proportioners. Carbon dioxide gas was used to control $\mathrm{pH}$ and nitrogen gas to control DO concentrations (Gobler et al. 2014). To produce diurnal changes in $\mathrm{pH}$ and DO concentrations, ITT Alcon solenoid valves were attached to the compressed gas tanks and ambient air lines, and were controlled with a Rain Bird timer. During the day cycle (09:00 to 21:00 h), the valves on the ambient airlines were opened and the valves on the other mixes of gas were closed to create control $\mathrm{pH}$ and DO conditions. At night (21:00 to 09:00 h), the valves on the appropriate $\mathrm{CO}_{2}, \mathrm{~N}_{2}$, or $\mathrm{CO}_{2}+\mathrm{N}_{2}$ gas tanks were opened to create low $\mathrm{pH}$ and/or DO conditions.

\section{Measuring environmental conditions}

Salinity was measured using a YSI 600QS multiparameter water quality sonde and temperature logged every $15 \mathrm{~min}$ on a HOBO U-002-64 data logger (Onset). The relative standard deviation of replicated temperature and salinity measurements was $<1 \%$, whereas the accuracy was $\pm 0.15^{\circ} \mathrm{C}$ and $0.1 \mathrm{psu}$, respectively. Daily measurements of $\mathrm{pH}$ were made with a Honeywell Durafet Ion Sensitive Field Effect Transistor (ISFET)-based pH sensor calibrated with a seawater pH standard (Dickson 1993) and logged every $15 \mathrm{~min}$ in the diurnal treatments with a 
Thermo-Scientific Orion STAR A321 pH meter. The relative standard deviation of replicated Durafet and Orion $\mathrm{pH}$ measurements was $<1 \%$, whereas the accuracy was \pm 0.005 and 0.002 units, respectively. A Clark-type electrode YSI 5100 oxygen meter was used to make daily DO measurements in all experimental vessels and DO was logged every 15 min on a HOBO U26 dissolved oxygen logger (Onset) in the diurnal treatments. The relative standard deviation of replicated dissolved oxygen measurements was $<1 \%$, whereas the accuracy was $<0.1 \%$. Previous studies have found that these instruments measure levels of DO that are indistinguishable from discrete measurements made with Winkler titrations (Gobler et al. 2014).

Dissolved inorganic carbon (DIC) measurements were made at the beginning and end of each experiment using a Liqui-Cel Membrane (Membrana) to separate the gaseous DIC from the seawater, which was then quantified with an EGM-4 Environmental Gas Analyzer (PP Systems) system. For all diurnal fluctuation treatments, samples were collected and analyzed from the end of both a day and night cycle. To determine the precision and accuracy of this technique, Dr. Andrew Dickson's (University of California San Diego, Scripps Institution of Oceanography) certified reference material for DIC was analyzed during each analytic run (percent recovery of DIC across all analytical runs: $103 \pm 6 \%$, mean \pm SD). DIC levels, along with $\mathrm{pH}$, temperature, salinity, pressure, phosphate, silicate, and carbonic acid dissociation constants recommended for estuarine waters (Millero 2010), were analyzed with the CO2SYS program (http://cdiac.ornl.gov/ftp/co2sys/) in order to quantify levels of $p \mathrm{CO}_{2}$, saturation states of calcite and aragonite $\left(\Omega_{\text {calciter }} \Omega_{\text {aragoniter }}\right.$ respectively), carbonate, and total alkalinity. Since $\mathrm{pH}$ values in some experimental treatments were made to fluctuate widely each day, and as $\mathrm{pH}$ is on a log scale, mean $\mathrm{pH}$ values were determined by first converting $\mathrm{pH}$ to $\left[\mathrm{H}^{+}\right]$ concentrations. Mean $\left[\mathrm{H}^{+}\right]$concentrations were then converted back to $\mathrm{pH}$.

\section{Larvae}

This study examined the effects of low $\mathrm{pH}$ and low DO on larval stage bay scallops Argopecten irradians, hard clams Mercenaria mercenaria, and eastern oysters Crassostrea virginica obtained from the East Hampton Town Shellfish Hatchery located in Montauk, NY, USA. Broodstock were collected from mesotrophic regions of eastern Long Island estuaries
(Shinnecock and Peconic Bays) in accordance with New York State Department of Environmental Conservation Collector's Permits. Adults were conditioned following the Food and Agriculture Organization of the United Nations' (FAO) protocol for shellfish aquaculture, being fed $\sim 3 \%$ of their dry weight in algae for 6 to $8 \mathrm{wk}$ and maintained at a temperature of $18^{\circ} \mathrm{C}$ (Helm et al. 2004). The algal diet included a mixture of the phytoplankton Isochrysis galbana, Tetraselmis suecica, T. chuii, Chaetoceros muelleri, C. calcitrans, and Pavlova lutheri in equal biovolumes (Helm et al. 2004). Adult shellfish were then temperature-spawned, and fertilized embryos were collected.

Each experimental vessel was stocked with 10000 D-stage larvae less than $24 \mathrm{~h}$ old. All larvae were fed a diet of $4 \times 10^{4}$ cells ml ${ }^{-1}$ of I. galbana daily (Carriker 2001, Helm et al. 2004, Cragg 2006). Full water changes were performed twice per week, during which all contents of each experimental vessel were poured through a $64 \mu \mathrm{m}$ sieve. Larvae collected on the sieve were condensed into a $50 \mathrm{ml}$ container from which $2 \mathrm{ml}$ were removed and preserved with a $3 \%$ solution of buffered formalin phosphate to assess mortality, size (distance from tip of the umbo to ventral side), and developmental stage (veliger, pediveliger, or metamorphosed) at each time point using a dissecting microscope with Nikon DigiSight Color Digital Camera System (DSVi1) and ImageJ software. Larvae that were alive at the time of preservation were counted to determine the rate of mortality in each treatment, and were distinguishable from dead larvae by pigmentation and morphology. Percent metamorphosis was calculated, based on the total number of surviving larvae at each time point, and larval experiments continued until all individuals in the control treatment had metamorphosed. Owing to the propensity of $C$. virginica larvae to set irreversibly on surfaces when metamorphosed, this experiment was ended after $14 \mathrm{~d}$, prior to the larvae metamorphosing into juveniles, and thus metamorphosis was not documented.

\section{Data analysis}

Statistical analyses were performed in RStudio. Survival and development data were arcsine square root transformed before analysis. Two-way ANOVAs were performed on the survival, development, and growth rate data, where $\mathrm{pH}$ and DO exposure levels (normal, chronically low, or diurnal) were the main treatment effects. While the experimental design was 
not fully balanced, all assumptions were met for these parametric tests. The Shapiro-Wilks test was used to assure all data were normally distributed and Bartlett's test was used to assure homogeneity of variance among each dataset. Two-way ANOVAs reporting significant effects from treatments were followed with Tukey's HSD test for all pairwise comparisons.

\section{RESULTS}

\section{Argopecten irradians (bay scallop) larvae}

There was a significant negative effect of $\mathrm{pH}$ (2-way ANOVA: $\mathrm{p}<0.001)$ and DO ( $<<0.001)$ on survival of Argopecten irradians larvae, and an interaction between the factors $(\mathrm{p}<0.05)$, with all manipulated conditions significantly reducing survival relative to the control condition (Table 1, Fig. 2). Percent survival for the control, low $\mathrm{pH}$, low $\mathrm{DO}$, low $\mathrm{pH}-\mathrm{DO}$, diurnal $\mathrm{pH}$, diurnal $\mathrm{DO}$, and diurnal $\mathrm{pH}-\mathrm{DO}$ conditions was $38 \pm 2,15 \pm 4,25 \pm 6,5 \pm 6,12 \pm 3$, $17 \pm 5$, and $7 \pm 2 \%$, respectively (Fig. 2 A). Survival under continuously low DO was higher than the low
$\mathrm{pH}(\mathrm{p}=0.006)$, diurnal $\mathrm{pH}(\mathrm{p}<0.001)$, and diurnal $\mathrm{pH}-\mathrm{DO}(\mathrm{p}<0.001)$ conditions, though still significantly lower than the control treatment $(p=0.009)$. The interaction between DO and $\mathrm{pH}$ was most apparent in the diurnal pH-DO treatment, where the survival $(7 \pm 2 \%)$ was higher than would have been predicted by the reductions in survival in the individual diurnal $\mathrm{pH}$ and diurnal DO treatments $(12 \pm 3$ and $17 \pm 5 \%$, respectively).

Growth rates of $A$. irradians larvae were affected by both $\mathrm{pH}(2$-way ANOVA: $\mathrm{p}<0.001)$ and DO ( $<<$ 0.001 ), but there was no interaction between the factors. Larvae experienced significantly slowed growth under all manipulated conditions, except for the diurnally fluctuating $\mathrm{pH}$ treatment (Fig. 2B). Control larvae grew at a rate of $13 \pm 1 \mu \mathrm{m} \mathrm{d}^{-1}$, while rates were slowed in the low $\mathrm{pH}-\mathrm{DO}(\mathrm{p}<0.001)$, low $\mathrm{pH}(\mathrm{p}<$ $0.001)$, diurnal $\mathrm{pH}-\mathrm{DO}(\mathrm{p}<0.001)$, diurnal DO $(\mathrm{p}=$ $0.018)$, and low DO $(\mathrm{p}=0.024)$ treatments. Both $\mathrm{pH}$ $(\mathrm{p}<0.001)$ and DO $(\mathrm{p}<0.001)$ slowed development of A. irradians larvae (2-way ANOVA). Fourteen days post-fertilization, $67 \pm 5 \%$ of larvae had metamorphosed under control conditions (Fig. 2C). Continuously low $\mathrm{pH}(\mathrm{p}<0.001)$ and continuously low DO $(p=0.026)$ significantly reduced metamorphosis

Table 1. Mean $( \pm \mathrm{SD}) \mathrm{pH}\left(\mathrm{pH}_{\mathrm{T}}=\mathrm{pH}\right.$ on the total scale), dissolved oxygen $(\mathrm{DO}), p \mathrm{CO}_{2}$, saturation states of calcite $\left(\Omega_{\text {calcite }}\right)$ and aragonite $\left(\Omega_{\text {aragonite }}\right)$, total dissolved inorganic carbon (DIC), carbonate, total alkalinity (TA), salinity, and temperature for larval Argopecten irradians diurnal acidification and hypoxia experiment. For diurnal treatments, conditions measured in the middle of the day and middle of the night cycles are depicted, along with a mean of the entire experiment

\begin{tabular}{|c|c|c|c|c|c|c|c|c|c|c|c|c|c|}
\hline \multirow[t]{2}{*}{ Parameter } & \multicolumn{4}{|c|}{$\longrightarrow$ Continuous -} & \multicolumn{3}{|c|}{ Diurnal pH } & \multicolumn{3}{|c|}{ - Diurnal DO } & \multicolumn{3}{|c|}{ —Diurnal pH-DO- } \\
\hline & Control & Low pH & Low DO & Low $\mathrm{pH}-\mathrm{DO}$ & Day & Night & Mean & Day & Night & Mean & Day & Night & Mean \\
\hline $\mathrm{pH}_{\mathrm{T}}$ & $\begin{array}{c}7.91 \\
\pm 0.02\end{array}$ & $\begin{array}{c}7.20 \\
\pm 0.09\end{array}$ & $\begin{array}{c}7.91 \\
\pm 0.02\end{array}$ & $\begin{array}{c}7.24 \\
\pm 0.11\end{array}$ & $\begin{array}{c}7.83 \\
\pm 0.13\end{array}$ & $\begin{array}{c}7.16 \\
\pm 0.11\end{array}$ & $\begin{array}{c}7.47 \\
\pm 0.23\end{array}$ & $\begin{array}{c}7.95 \\
\pm 0.06\end{array}$ & $\begin{array}{l}7.95 \\
\pm 0.06\end{array}$ & $\begin{array}{c}7.90 \\
\pm 0.02\end{array}$ & $\begin{array}{c}7.90 \\
\pm 0.06\end{array}$ & $\begin{array}{l}7.28 \\
\pm 0.08\end{array}$ & $\begin{array}{c}7.58 \\
\pm 0.25\end{array}$ \\
\hline $\mathrm{DO}\left(\mathrm{mg} \mathrm{l}^{-1}\right)$ & $\begin{array}{c}6.87 \\
\pm 0.33\end{array}$ & $\begin{array}{c}6.96 \\
\pm 0.74\end{array}$ & $\begin{array}{c}2.33 \\
\pm 0.64\end{array}$ & $\begin{array}{c}2.19 \\
\pm 0.92\end{array}$ & $\begin{array}{c}6.84 \\
\pm 0.19\end{array}$ & $\begin{array}{c}6.77 \\
\pm 0.53\end{array}$ & $\begin{array}{c}6.80 \\
\pm 0.57\end{array}$ & $\begin{array}{c}6.82 \\
\pm 1.70\end{array}$ & $\begin{array}{c}1.43 \\
\pm 0.34\end{array}$ & $\begin{array}{c}4.24 \\
\pm 2.80\end{array}$ & $\begin{array}{c}7.24 \\
\pm 0.12\end{array}$ & $\begin{array}{c}1.30 \\
\pm 0.28\end{array}$ & $\begin{array}{c}4.28 \\
\pm 2.90\end{array}$ \\
\hline$p \mathrm{CO}_{2}(\mu \mathrm{atm})$ & $\begin{array}{c}516 \\
\pm 9.0\end{array}$ & $\begin{array}{r}3480 \\
\pm 190\end{array}$ & $\begin{array}{l}548 \\
\pm 56\end{array}$ & $\begin{array}{r}3290 \\
\pm 234\end{array}$ & $\begin{array}{r}586 \\
\pm 37\end{array}$ & $\begin{array}{r}3180 \\
\pm 181\end{array}$ & $\begin{array}{c}1880 \\
\pm 1390\end{array}$ & $\begin{array}{l}573 \\
\pm 91\end{array}$ & $\begin{array}{r}515 \\
\pm 21\end{array}$ & $\begin{array}{l}544 \\
\pm 69\end{array}$ & $\begin{array}{r}623 \\
\pm 41\end{array}$ & $\begin{array}{r}2930 \\
\pm 241\end{array}$ & $\begin{array}{c}1770 \\
\pm 1250\end{array}$ \\
\hline$\Omega_{\text {calcite }}$ & $\begin{array}{c}3.00 \\
\pm 0.12\end{array}$ & $\begin{array}{c}0.60 \\
\pm 0.07\end{array}$ & $\begin{array}{c}2.93 \\
\pm 0.23\end{array}$ & $\begin{array}{c}0.61 \\
\pm 0.08\end{array}$ & $\begin{array}{c}2.61 \\
\pm 0.11\end{array}$ & $\begin{array}{c}0.65 \\
\pm 0.11\end{array}$ & $\begin{array}{c}1.63 \\
\pm 1.10\end{array}$ & $\begin{array}{c}2.78 \\
\pm 0.09\end{array}$ & $\begin{array}{c}2.80 \\
\pm 0.12\end{array}$ & $\begin{array}{c}2.79 \\
\pm 0.10\end{array}$ & $\begin{array}{c}2.48 \\
\pm 0.02\end{array}$ & $\begin{array}{c}0.69 \\
\pm 0.13\end{array}$ & $\begin{array}{c}1.59 \\
\pm 0.96\end{array}$ \\
\hline$\Omega_{\text {aragonite }}$ & $\begin{array}{c}1.94 \\
\pm 0.08\end{array}$ & $\begin{array}{c}0.39 \\
\pm 0.05\end{array}$ & $\begin{array}{c}1.90 \\
\pm 0.15\end{array}$ & $\begin{array}{c}0.39 \\
\pm 0.05\end{array}$ & $\begin{array}{c}1.69 \\
\pm 0.07\end{array}$ & $\begin{array}{c}0.42 \\
\pm 0.07\end{array}$ & $\begin{array}{c}1.05 \\
\pm 0.68\end{array}$ & $\begin{array}{l}1.79 \\
\pm 0.07\end{array}$ & $\begin{array}{c}1.81 \\
\pm 0.08\end{array}$ & $\begin{array}{c}1.80 \\
\pm 0.07\end{array}$ & $\begin{array}{l}1.60 \\
\pm 0.02\end{array}$ & $\begin{array}{c}0.45 \\
\pm 0.09\end{array}$ & $\begin{array}{c}1.03 \\
\pm 0.62\end{array}$ \\
\hline $\mathrm{DIC}\left(\mu \mathrm{mol} \mathrm{l}{ }^{-1}\right)$ & $\begin{array}{l}1800 \\
\pm 31\end{array}$ & $\begin{array}{l}2060 \\
\pm 147\end{array}$ & $\begin{array}{l}1830 \\
\pm 91\end{array}$ & $\begin{array}{l}2010 \\
\pm 40\end{array}$ & $\begin{array}{l}1770 \\
\pm 16\end{array}$ & $\begin{array}{l}2050 \\
\pm 129\end{array}$ & $\begin{array}{r}1910 \\
\pm 169\end{array}$ & $\begin{array}{r}1810 \\
\pm 117\end{array}$ & $\begin{array}{l}1730 \\
\pm 31\end{array}$ & $\begin{array}{l}1770 \\
\pm 55\end{array}$ & $\begin{array}{l}1770 \\
\pm 45\end{array}$ & $\begin{array}{l}2010 \\
\pm 140\end{array}$ & $\begin{array}{r}1890 \\
\pm 169\end{array}$ \\
\hline $\mathrm{CO}_{3}{ }^{2-}\left(\mu \mathrm{mol} \mathrm{l}^{-1}\right)$ & $\begin{array}{c}120 \\
\pm 5.0\end{array}$ & $\begin{array}{r}24.0 \\
\pm 3.0\end{array}$ & $\begin{array}{c}117 \\
\pm 9.4\end{array}$ & $\begin{array}{r}24.3 \\
\pm 3.2\end{array}$ & $\begin{array}{c}104 \\
\pm 4.4\end{array}$ & $\begin{array}{r}26.2 \\
\pm 4.5\end{array}$ & $\begin{array}{l}65.2 \\
\pm 55\end{array}$ & $\begin{array}{c}111 \\
\pm 4.3\end{array}$ & $\begin{array}{c}112 \\
\pm 5.4\end{array}$ & $\begin{array}{c}111 \\
\pm 0.57\end{array}$ & $\begin{array}{r}99.1 \\
\pm 1.5\end{array}$ & $\begin{array}{r}27.7 \\
\pm 5.4\end{array}$ & $\begin{array}{l}63.4 \\
\pm 51\end{array}$ \\
\hline TA $\left(\mu \mathrm{mol} \mathrm{l} 1^{-1}\right)$ & $\begin{array}{l}1970 \\
\pm 36\end{array}$ & $\begin{array}{r}1990 \\
\pm 147\end{array}$ & $\begin{array}{l}1990 \\
\pm 93\end{array}$ & $\begin{array}{l}1950 \\
\pm 52\end{array}$ & $\begin{array}{l}1920 \\
\pm 15\end{array}$ & $\begin{array}{r}1990 \\
\pm 137\end{array}$ & $\begin{array}{l}1950 \\
\pm 53\end{array}$ & $\begin{array}{r}1960 \\
\pm 104\end{array}$ & $\begin{array}{l}1890 \\
\pm 34\end{array}$ & $\begin{array}{l}1930 \\
\pm 51\end{array}$ & $\begin{array}{l}1910 \\
\pm 40\end{array}$ & $\begin{array}{c}1960 \\
\pm 150\end{array}$ & $\begin{array}{l}1940 \\
\pm 42\end{array}$ \\
\hline Salinity & $\begin{array}{l}29.5 \\
\pm 0.6\end{array}$ & $\begin{array}{l}29.5 \\
\pm 0.6\end{array}$ & $\begin{array}{l}29.5 \\
\pm 0.6\end{array}$ & $\begin{array}{l}29.5 \\
\pm 0.6\end{array}$ & $\begin{array}{r}29.5 \\
\pm 0.6\end{array}$ & $\begin{array}{l}29.5 \\
\pm 0.6\end{array}$ & $\begin{array}{r}29.5 \\
\pm 0.6\end{array}$ & $\begin{array}{l}29.5 \\
\pm 0.6\end{array}$ & $\begin{array}{l}29.5 \\
\pm 0.6\end{array}$ & $\begin{array}{l}29.5 \\
\pm 0.6\end{array}$ & $\begin{array}{r}29.5 \\
\pm 0.6\end{array}$ & $\begin{array}{l}29.5 \\
\pm 0.6\end{array}$ & $\begin{array}{l}29.5 \\
\pm 0.6\end{array}$ \\
\hline $\begin{array}{l}\text { Temperature } \\
\left({ }^{\circ} \mathrm{C}\right)\end{array}$ & $\begin{array}{l}23.1 \\
\pm 0.3\end{array}$ & $\begin{array}{l}23.1 \\
\pm 0.3\end{array}$ & $\begin{array}{l}23.1 \\
\pm 0.3\end{array}$ & $\begin{array}{l}23.1 \\
\pm 0.3\end{array}$ & $\begin{array}{l}23.1 \\
\pm 0.3\end{array}$ & $\begin{array}{l}23.1 \\
\pm 0.3\end{array}$ & $\begin{array}{c}23.1 \\
\pm 0.3\end{array}$ & $\begin{array}{l}23.1 \\
\pm 0.3\end{array}$ & $\begin{array}{l}23.1 \\
\pm 0.3\end{array}$ & $\begin{array}{l}23.1 \\
\pm 0.3\end{array}$ & $\begin{array}{l}23.1 \\
\pm 0.3\end{array}$ & $\begin{array}{l}23.1 \\
\pm 0.3\end{array}$ & $\begin{array}{l}23.1 \\
\pm 0.3\end{array}$ \\
\hline
\end{tabular}



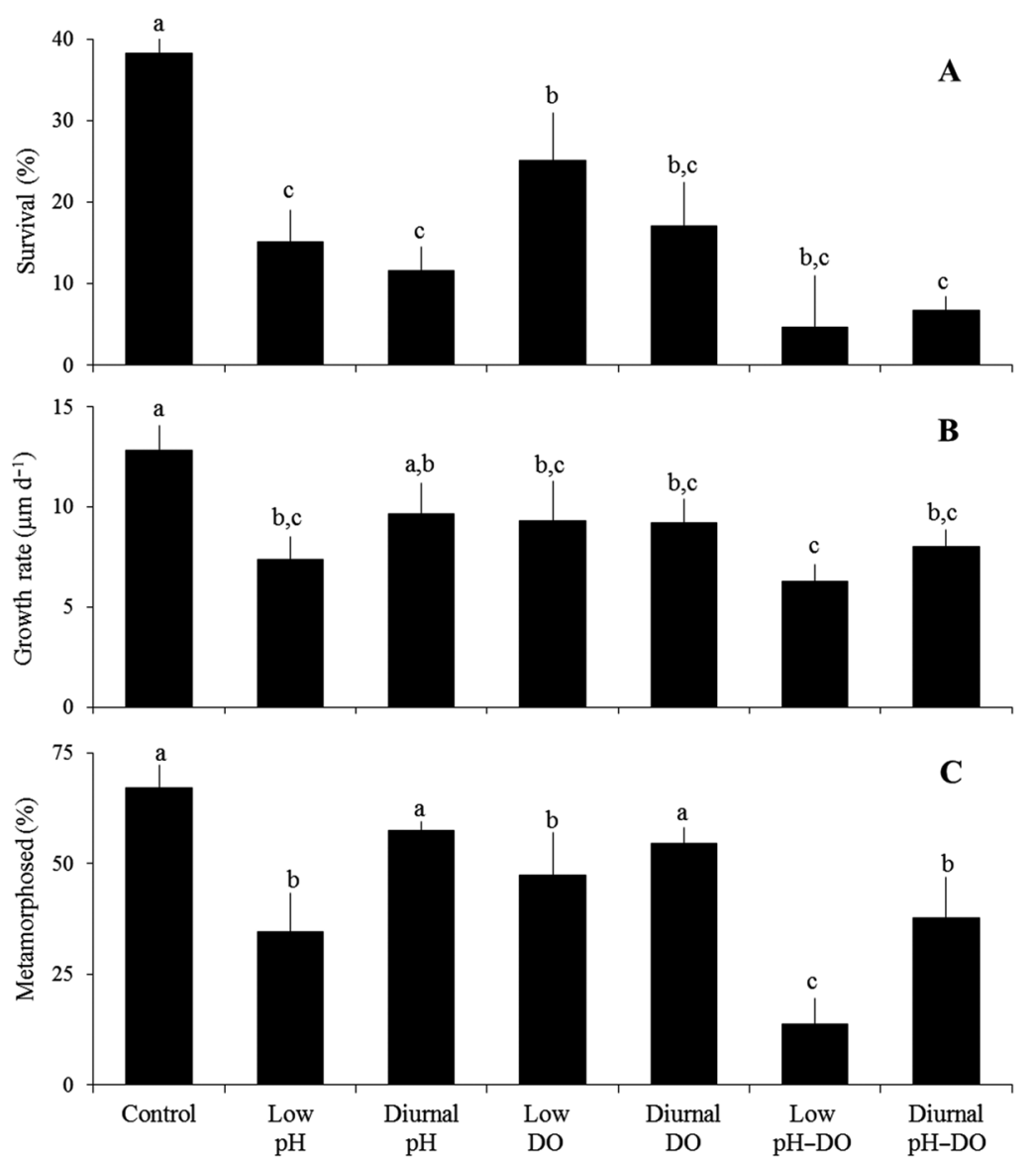

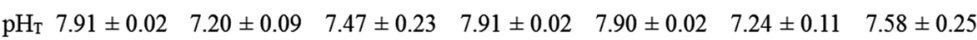

$\mathrm{DO}\left(\mathrm{mg} \mathrm{l}^{-1}\right) 6.87 \pm 0.33 \quad 6.96 \pm 0.74 \quad 6.80 \pm 0.57 \quad 2.33 \pm 0.64 \quad 4.24 \pm 2.80 \quad 2.19 \pm 0.92 \quad 4.28 \pm 2.90$

Fig. 2. (A) Survival, (B) growth, and (C) development of Argopecten irradians larvae. Percent metamorphosis was calculated $14 \mathrm{~d}$ post-fertilization. Error bars represent SD of the mean $(\mathrm{n}=4)$. Mean $\mathrm{pH}$ and DO values during the experiment for each treatment are shown, whereas detailed experimental conditions appear in Table 1. Letters above bars denote significant groupings of treatments as indicated via post hoc Tukey's HSD tests

to $35 \pm 9$ and $48 \pm 10 \%$, respectively, but diurnal exposure to low $\mathrm{pH}$ and to low DO did not alter the fraction of larvae that had metamorphosed. Metamorphosis was significantly delayed in the low $\mathrm{pH}$-DO treatment $(\mathrm{p}<0.001)$ to $14 \pm 6 \%$, and to a significantly lesser extent in the diurnal $\mathrm{pH}-\mathrm{DO}$ treatment $(38 \pm 9 \%, \mathrm{p}<0.001)$.

\section{Mercenaria mercenaria (hard clam) larvae}

Survival of larval Mercenaria mercenaria was significantly reduced by $\mathrm{pH}$ (2-way ANOVA: $\mathrm{p}<0.001)$ but not $\mathrm{DO}$, and there was an interactive effect of these 2 factors ( $p<0.05$; Table 2, Fig. 3). Under control, chronically low DO, and fluctuating low DO con- ditions, $28 \pm 2,24 \pm 3$, and $27 \pm 5 \%$ of larvae, respectively, survived to the end of the experiment, while survival was significantly reduced to $1 \pm 0.4,2 \pm 1,1 \pm 1$, and $5 \pm 3 \%$ in the low $\mathrm{pH}(\mathrm{p}<0.001)$, diurnal $\mathrm{pH}(\mathrm{p}<0.001)$, low $\mathrm{pH}-\mathrm{DO}(\mathrm{p}<0.001)$, and diurnal $\mathrm{pH}-\mathrm{DO}(\mathrm{p}<0.001)$ treatments, respectively (Fig. 3A). There was an interaction between $\mathrm{pH}$ and $\mathrm{DO}$ as the survival in the combined diurnal treatment was higher than would have been predicted by the individual treatments for both the static and diurnal treatments.

There was an effect of $\mathrm{pH}$ (2-way ANOVA: $\mathrm{p}<0.05)$ and DO $(\mathrm{p}<0.05)$ on the growth of $M$. mercenaria larvae, although post hoc multiple comparisons did not reveal differences among treatments (Fig. 3B). Development of $M$. mercenaria larvae was affected by $\mathrm{pH}$ (2-way ANOVA: $\mathrm{p}<0.001)$ and DO ( $<<0.001)$, and there was an interaction between these 2 factors $(p<0.001)$. Fewer larvae metamorphosed 17 d postfertilization in all $\mathrm{pH}$ and DO treatments compared to $80 \pm 6 \%$ that had metamorphosed in control conditions $(\mathrm{p}<0.001$; Fig. 3C). There was an interactive effect of $\mathrm{pH}$ and DO on development, with $21 \pm 6$ and $31 \pm 7 \%$ of larvae reaching metamorphosis in the chronically low $\mathrm{pH}(\mathrm{p}<0.001)$ and chronically low DO $(\mathrm{p}<0.001)$ treatments, respectively, and $7 \pm 2 \%$ in the chronically low $\mathrm{pH}-\mathrm{DO}(\mathrm{p}<0.001)$ treatment, a value higher than would have been predicted by the individual treatments. Although fewer larvae developed to metamorphosis in the low DO and diurnal DO $(25 \pm 5 \%, p<0.001$ for both) treatments than in the control, there were significantly more metamorphosed larvae in these 2 treatments than in the diurnal $\mathrm{pH}(9 \pm 4 \%, \mathrm{p}<0.001, \mathrm{p}=0.002)$, low $\mathrm{pH}-\mathrm{DO}$ $(7 \pm 2 \%, \mathrm{p}<0.001, \mathrm{p}<0.001)$, and diurnal $\mathrm{pH}-\mathrm{DO}$ $(11 \pm 3 \%, p=0.001, p=0.034)$ treatments.

\section{Crassostrea virginica (eastern oyster) larvae}

DO and $\mathrm{pH}$ significantly reduced the survival of Crassostrea virginica larvae (2-way ANOVA: $\mathrm{p}<0.05$ and $\mathrm{p}<0.001$, respectively) and there was no interaction between these factors (Table 3, Fig. 4). Survival was reduced from $15 \pm 5 \%$ under control conditions to $3 \pm 1,5 \pm 4$, and $5 \pm 2 \%$ in low $\mathrm{pH}(\mathrm{p}<0.001)$, low 
Fig. 3. (A) Survival, (B) growth, and (C) development of Mercenaria mercenaria larvae. Percent metamorphosis was calculated $17 \mathrm{~d}$ post-fertilization. Error bars represent SD of the mean $(n=4)$. Mean $\mathrm{pH}$ and DO values during the experiment for each treatment are shown, whereas detailed experimental conditions appear in Table 2. Letters above bars denote significant groupings of treatments as indicated via post hoc Tukey's HSD tests

$\mathrm{pH}-\mathrm{DO}(\mathrm{p}=0.015)$, and diurnal $\mathrm{pH}-\mathrm{DO}(\mathrm{p}=$ 0.017 ) treatments, respectively (Fig. 4A). There were no significant survival differences between control conditions and the treatment with diurnal fluctuations in $\mathrm{pH}$ or between diurnal and continuous low DO treatment: these survival rates were all significantly higher than in the low $\mathrm{pH}$, low $\mathrm{pH}-\mathrm{DO}$, and diurnal $\mathrm{pH}-\mathrm{DO}$ treatments ( $\mathrm{p}<0.05$ for all). Finally, the percent survival of $C$. virginica larvae was significantly higher in the low DO treatment than in the diurnal $\mathrm{pH}$ treatment $(p=0.002)$.

There was an effect of $\mathrm{pH}$ (2-way ANOVA: $p<0.001)$, DO $(p<0.05)$, and an interactive effect of $\mathrm{pH}$ and DO $(\mathrm{p}<0.05)$ on the growth rates of $C$. virginica larvae. Growth rates were reduced from $1 \pm 0.1 \mu \mathrm{m} \mathrm{d}^{-1}$ in control conditions to $0.7 \pm 0.1,0.3 \pm 0.2,0.2 \pm 0.1$
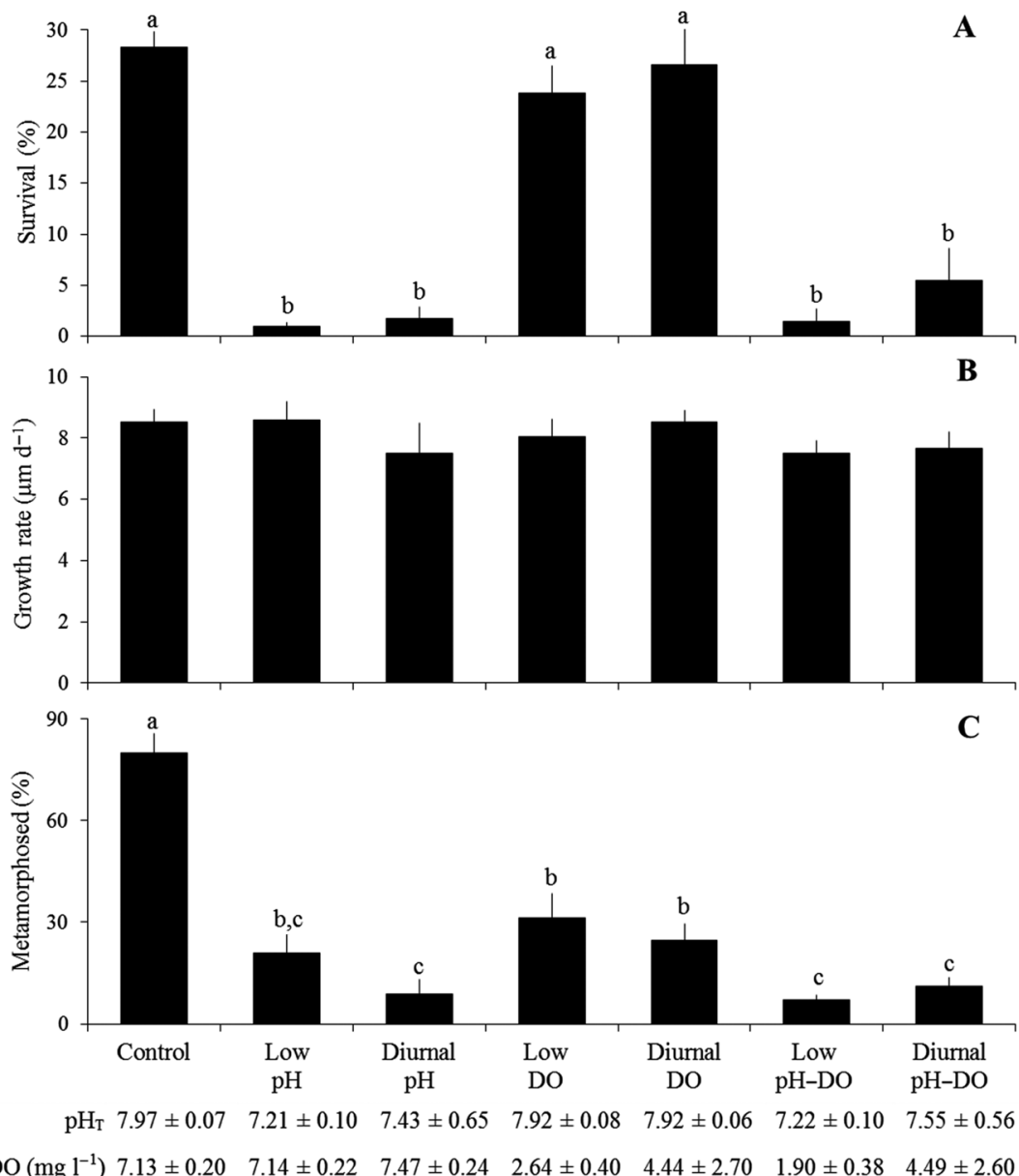

Table 2. Mean ( $\pm \mathrm{SD}$ ) $\mathrm{pH}, \mathrm{DO}, p \mathrm{CO}_{2}, \Omega_{\text {calcite, }} \Omega_{\text {aragonite, }}$ DIC, carbonate, TA, salinity, and temperature for larval Mercenaria mercenaria diurnal acidification and hypoxia experiment. For diurnal treatments, conditions measured in the middle of the day and middle of the night cycles are depicted, along with a mean of the entire experiment

\begin{tabular}{|c|c|c|c|c|c|c|c|c|c|c|c|c|c|}
\hline \multirow[t]{2}{*}{ Parameter } & \multicolumn{4}{|c|}{ Continuous } & \multicolumn{3}{|c|}{$\ldots$ Diurnal pH } & \multicolumn{3}{|c|}{${ }_{-}$Diurnal DO } & \multicolumn{3}{|c|}{ _ Diurnal pH-DO _ } \\
\hline & Control & Low $\mathrm{pH}$ & Low DO & Low pH-DO & Day & Night & Mean & Day & Night & Mean & Day & Night & Mean \\
\hline $\mathrm{pH}_{\mathrm{T}}$ & $\begin{array}{c}7.97 \\
\pm 0.07\end{array}$ & $\begin{array}{c}7.21 \\
\pm 0.10\end{array}$ & $\begin{array}{c}7.92 \\
\pm 0.08\end{array}$ & $\begin{array}{c}7.22 \\
\pm 0.10\end{array}$ & $\begin{array}{c}7.94 \\
\pm 0.03\end{array}$ & $\begin{array}{c}7.16 \\
\pm 0.07\end{array}$ & $\begin{array}{c}7.43 \\
\pm 0.65\end{array}$ & $\begin{array}{c}7.93 \\
\pm 0.04\end{array}$ & $\begin{array}{c}7.92 \\
\pm 0.10\end{array}$ & $\begin{array}{c}7.92 \\
\pm 0.06\end{array}$ & $\begin{array}{c}7.95 \\
\pm 0.10\end{array}$ & $\begin{array}{c}7.29 \\
\pm 0.06\end{array}$ & $\begin{array}{c}7.55 \\
\pm 0.56\end{array}$ \\
\hline $\mathrm{DO}\left(\mathrm{mg} \mathrm{l}^{-1}\right)$ & $\begin{array}{c}7.13 \\
\pm 0.20\end{array}$ & $\begin{array}{c}7.14 \\
\pm 0.22\end{array}$ & $\begin{array}{c}2.64 \\
\pm 0.40\end{array}$ & $\begin{array}{c}1.90 \\
\pm 0.38\end{array}$ & $\begin{array}{c}7.49 \\
\pm 0.18\end{array}$ & $\begin{array}{c}7.51 \\
\pm 0.33\end{array}$ & $\begin{array}{c}7.47 \\
\pm 0.24\end{array}$ & $\begin{array}{c}7.35 \\
\pm 0.24\end{array}$ & $\begin{array}{c}1.59 \\
\pm 0.38\end{array}$ & $\begin{array}{c}4.44 \\
\pm 2.70\end{array}$ & $\begin{array}{c}7.32 \\
\pm 0.29\end{array}$ & $\begin{array}{c}1.58 \\
\pm 0.37\end{array}$ & $\begin{array}{c}4.49 \\
\pm 2.60\end{array}$ \\
\hline$p \mathrm{CO}_{2}(\mu \mathrm{atm})$ & $\begin{array}{l}509 \\
\pm 70\end{array}$ & $\begin{array}{l}3580 \\
\pm 70\end{array}$ & $\begin{array}{c}521 \\
\pm 62\end{array}$ & $\begin{array}{l}3100 \\
\pm 61\end{array}$ & $\begin{array}{c}601 \\
\pm 54\end{array}$ & $\begin{array}{l}3130 \\
\pm 268\end{array}$ & $\begin{array}{c}1870 \\
\pm 1470\end{array}$ & $\begin{array}{c}500 \\
\pm 9.2\end{array}$ & $\begin{array}{c}507 \pm 9.2 \\
\pm 9.2\end{array}$ & $\begin{array}{c}504 \\
\pm 6.5\end{array}$ & $\begin{array}{l}601 \\
\pm 54\end{array}$ & $\begin{array}{l}2860 \\
\pm 118\end{array}$ & $\begin{array}{c}1730 \\
\pm 1310\end{array}$ \\
\hline$\Omega_{\text {calcite }}$ & $\begin{array}{c}3.04 \\
\pm 0.05\end{array}$ & $\begin{array}{c}0.66 \\
\pm 0.01\end{array}$ & $\begin{array}{c}3.03 \\
\pm 0.16\end{array}$ & $\begin{array}{c}0.67 \\
\pm 0.01\end{array}$ & $\begin{array}{c}2.60 \\
\pm 0.19\end{array}$ & $\begin{array}{c}0.74 \\
\pm 0.06\end{array}$ & $\begin{array}{c}1.67 \\
\pm 1.08\end{array}$ & $\begin{array}{c}2.85 \\
\pm 0.04\end{array}$ & $\begin{array}{c}2.89 \\
\pm 0.08\end{array}$ & $\begin{array}{c}2.87 \\
\pm 0.06\end{array}$ & $\begin{array}{c}2.50 \\
\pm 0.00\end{array}$ & $\begin{array}{c}0.80 \\
\pm 0.02\end{array}$ & $\begin{array}{c}1.65 \\
\pm 0.98\end{array}$ \\
\hline$\Omega_{\text {aragonite }}$ & $\begin{array}{c}1.97 \\
\pm 0.03\end{array}$ & $\begin{array}{c}0.43 \\
\pm 0.01\end{array}$ & $\begin{array}{c}1.97 \\
\pm 0.01\end{array}$ & $\begin{array}{c}0.44 \\
\pm 0.01\end{array}$ & $\begin{array}{c}1.68 \\
\pm 0.12\end{array}$ & $\begin{array}{c}0.48 \\
\pm 0.04\end{array}$ & $\begin{array}{c}1.08 \\
\pm 0.70\end{array}$ & $\begin{array}{c}1.85 \\
\pm 0.03\end{array}$ & $\begin{array}{c}1.87 \\
\pm 0.05\end{array}$ & $\begin{array}{c}1.86 \\
\pm 0.04\end{array}$ & $\begin{array}{c}1.62 \\
\pm 0.00\end{array}$ & $\begin{array}{c}0.52 \\
\pm 0.01\end{array}$ & $\begin{array}{c}1.07 \\
\pm 0.64\end{array}$ \\
\hline DIC $\left(\mu \mathrm{mol} \mathrm{l}{ }^{-1}\right)$ & $\begin{array}{c}1790 \\
\pm 0.56\end{array}$ & $\begin{array}{l}2180 \\
\pm 43\end{array}$ & $\begin{array}{r}1810 \\
\pm 152\end{array}$ & $\begin{array}{l}2040 \\
\pm 8.3\end{array}$ & $\begin{array}{r}1780 \\
\pm 7.5\end{array}$ & $\begin{array}{l}2160 \\
\pm 14\end{array}$ & $\begin{array}{l}1970 \\
\pm 216\end{array}$ & $\begin{array}{l}1720 \\
\pm 2.0\end{array}$ & $\begin{array}{l}17401 \\
\pm 24\end{array}$ & $\begin{array}{c}1730 \pm 24 \\
\quad \pm 24\end{array}$ & $\begin{array}{l}1740 \\
\pm 2.1\end{array}$ & $\begin{array}{l}2130 \\
\pm 21\end{array}$ & $\begin{array}{r}1930 \\
\pm 281\end{array}$ \\
\hline $\mathrm{CO}_{3}{ }^{2-}\left(\mu \mathrm{mol} \mathrm{l}{ }^{-1}\right)$ & $\begin{array}{r}122 \\
\pm 1.9\end{array}$ & $\begin{array}{c}26.5 \\
\pm 0.52\end{array}$ & $\begin{array}{c}122 \\
\pm 6.5\end{array}$ & $\begin{array}{c}27.0 \\
\pm 0.34\end{array}$ & $\begin{array}{c}104 \\
\pm 7.6\end{array}$ & $\begin{array}{r}29.9 \\
\pm 2.3\end{array}$ & $\begin{array}{l}67.1 \\
\pm 53\end{array}$ & $\begin{array}{r}115 \\
\pm 1.6\end{array}$ & $\begin{array}{c}116 \\
\pm 3.4\end{array}$ & $\begin{array}{r}115 \\
\pm 1.0\end{array}$ & $\begin{array}{c}100 \\
\pm 0.12\end{array}$ & $\begin{array}{c}32.1 \\
\pm 0.76\end{array}$ & $\begin{array}{l}66.2 \\
\pm 48\end{array}$ \\
\hline $\mathrm{TA}\left(\mu \mathrm{mol} \mathrm{l} \mathrm{l}^{-1}\right)$ & $\begin{array}{l}1960 \\
\pm 3.6\end{array}$ & $\begin{array}{l}2110 \\
\pm 41\end{array}$ & $\begin{array}{r}1980 \\
\pm 155\end{array}$ & $\begin{array}{l}1990 \\
\pm 5.9\end{array}$ & $\begin{array}{r}1920 \\
\pm 5.6\end{array}$ & $\begin{array}{l}2110 \\
\pm 2.8\end{array}$ & $\begin{array}{l}2020 \\
\pm 128\end{array}$ & $\begin{array}{r}1880 \\
\pm 0.77\end{array}$ & $\begin{array}{l}1910 \\
\pm 28\end{array}$ & $\begin{array}{l}1890 \\
\pm 16\end{array}$ & $\begin{array}{l}1870 \\
\pm 2.2\end{array}$ & $\begin{array}{l}2100 \\
\pm 16\end{array}$ & $\begin{array}{r}1980 \\
\pm 157\end{array}$ \\
\hline Salinity & $\begin{array}{r}29.2 \\
\pm 0.8\end{array}$ & $\begin{array}{r}29.2 \\
\pm 0.8\end{array}$ & $\begin{array}{r}29.2 \\
\pm 0.8\end{array}$ & $\begin{array}{r}29.2 \\
\pm 0.8\end{array}$ & $\begin{array}{r}29.2 \\
\pm 0.8\end{array}$ & $\begin{array}{r}29.2 \\
\pm 0.8\end{array}$ & $\begin{array}{r}29.2 \\
\pm 0.8\end{array}$ & $\begin{array}{r}29.2 \\
\pm 0.8\end{array}$ & $\begin{array}{l}29.2 \\
\pm 0.8\end{array}$ & $\begin{array}{r}29.2 \\
\pm 0.8\end{array}$ & $\begin{array}{r}29.2 \\
\pm 0.8\end{array}$ & $\begin{array}{l}29.2 \\
\pm 0.8\end{array}$ & $\begin{array}{r}29.2 \\
\pm 0.8\end{array}$ \\
\hline $\begin{array}{l}\text { Temperature } \\
\left({ }^{\circ} \mathrm{C}\right)\end{array}$ & $\begin{array}{r}22.8 \\
\pm 0.2\end{array}$ & $\begin{array}{r}22.8 \\
\pm 0.2\end{array}$ & $\begin{array}{r}22.8 \\
\pm 0.2\end{array}$ & $\begin{array}{r}22.8 \\
\pm 0.2\end{array}$ & $\begin{array}{r}22.8 \\
\pm 0.2\end{array}$ & $\begin{array}{r}22.8 \\
\pm 0.2\end{array}$ & $\begin{array}{r}22.8 \\
\pm 0.2\end{array}$ & $\begin{array}{r}22.8 \\
\pm 0.2\end{array}$ & $\begin{array}{c}22.8 \\
\pm 0.21\end{array}$ & $\begin{array}{r}22.8 \\
\pm 0.2\end{array}$ & $\begin{array}{l}22.8 \\
\pm 0.2\end{array}$ & $\begin{array}{c}22.8 \\
\pm 0.21\end{array}$ & $\begin{array}{r}22.8 \\
\pm 0.2\end{array}$ \\
\hline
\end{tabular}


Table 3. Mean ( $\pm \mathrm{SD}) \mathrm{pH}, \mathrm{DO}, p \mathrm{CO}_{2}, \Omega_{\text {calcite, }} \Omega_{\text {aragonite, }}$ DIC, carbonate, TA, salinity, and temperature for larval Crassostrea virginica diurnal acidification and hypoxia experiment. For diurnal treatments, conditions measured in the middle of the day and middle of the night cycles are depicted, along with a mean of the entire experiment

\begin{tabular}{|c|c|c|c|c|c|c|c|c|c|c|c|c|c|}
\hline \multirow[t]{2}{*}{ Parameter } & \multicolumn{4}{|c|}{ Continuous - } & \multicolumn{3}{|c|}{$\ldots$ Diurnal pH } & \multicolumn{3}{|c|}{${ }_{-}$Diurnal DO__ } & \multicolumn{3}{|c|}{ — Diurnal pH-DO _ } \\
\hline & Control & Low $\mathrm{pH}$ & Low DO & Low pH-DO & Day & Night & Mean & Day & Night & Mean & Day & Night & Mean \\
\hline $\mathrm{pH}_{\mathrm{T}}$ & $\begin{array}{c}7.85 \\
\pm 0.04\end{array}$ & $\begin{array}{c}7.16 \\
\pm 0.07\end{array}$ & $\begin{array}{c}7.83 \\
\pm 0.05\end{array}$ & $\begin{array}{c}7.18 \\
\pm 0.07\end{array}$ & $\begin{array}{c}7.95 \\
\pm 0.03\end{array}$ & $\begin{array}{c}7.28 \\
\pm 0.06\end{array}$ & $\begin{array}{c}7.54 \\
\pm 0.26\end{array}$ & $\begin{array}{c}8.02 \\
\pm 0.04\end{array}$ & $\begin{array}{c}7.96 \\
\pm 0.12\end{array}$ & $\begin{array}{c}7.99 \\
\pm 0.09\end{array}$ & $\begin{array}{c}7.93 \\
\pm 0.04\end{array}$ & $\begin{array}{c}7.27 \\
\pm 0.03\end{array}$ & $\begin{array}{c}7.50 \\
\pm 0.23\end{array}$ \\
\hline $\mathrm{DO}\left(\mathrm{mg} \mathrm{l}^{-1}\right)$ & $\begin{array}{c}7.04 \\
\pm 0.16\end{array}$ & $\begin{array}{c}6.98 \\
\pm 0.16\end{array}$ & $\begin{array}{c}2.50 \\
\pm 0.71\end{array}$ & $\begin{array}{c}1.87 \\
\pm 0.41\end{array}$ & $\begin{array}{c}7.52 \\
\pm 0.13\end{array}$ & $\begin{array}{c}7.59 \\
\pm 0.24\end{array}$ & $\begin{array}{c}7.54 \\
\pm 0.18\end{array}$ & $\begin{array}{c}7.24 \\
\pm 0.12\end{array}$ & $\begin{array}{c}2.91 \\
\pm 1.50\end{array}$ & $\begin{array}{c}5.14 \\
\pm 2.09\end{array}$ & $\begin{array}{c}7.33 \\
\pm 0.05\end{array}$ & $\begin{array}{c}1.66 \\
\pm 0.56\end{array}$ & $\begin{array}{c}4.36 \\
\pm 2.79\end{array}$ \\
\hline$p \mathrm{CO}_{2}(\mu \mathrm{atm})$ & $\begin{array}{c}522 \\
\pm 2.7\end{array}$ & $\begin{array}{l}3380 \\
\pm 250\end{array}$ & $\begin{array}{r}575 \\
\pm 52\end{array}$ & $\begin{array}{l}3480 \\
\pm 134\end{array}$ & $\begin{array}{r}570 \\
\pm 16\end{array}$ & $\begin{array}{r}3230 \\
\pm 131\end{array}$ & $\begin{array}{c}1900 \\
\pm 1540\end{array}$ & $\begin{array}{r}645 \\
\pm 64\end{array}$ & $\begin{array}{c}524 \\
\pm 31\end{array}$ & $\begin{array}{r}585 \\
\pm 81\end{array}$ & $\begin{array}{c}644 \\
\pm 16\end{array}$ & $\begin{array}{c}2990 \\
\pm 380\end{array}$ & $\begin{array}{r}1820 \\
\pm 1370\end{array}$ \\
\hline$\Omega_{\text {calcite }}$ & $\begin{array}{c}2.96 \\
\pm 0.18\end{array}$ & $\begin{array}{c}0.54 \\
\pm 0.01\end{array}$ & $\begin{array}{c}2.83 \\
\pm 0.29\end{array}$ & $\begin{array}{c}0.54 \\
\pm 0.01\end{array}$ & $\begin{array}{c}2.62 \\
\pm 0.01\end{array}$ & $\begin{array}{c}0.56 \\
\pm 0.00\end{array}$ & $\begin{array}{r}1.59 \\
\pm 1.2\end{array}$ & $\begin{array}{c}2.70 \\
\pm 0.00\end{array}$ & $\begin{array}{c}2.70 \\
\pm 0.01\end{array}$ & $\begin{array}{c}2.70 \\
\pm 0.01\end{array}$ & $\begin{array}{c}2.46 \\
\pm 0.02\end{array}$ & $\begin{array}{c}0.59 \\
\pm 0.08\end{array}$ & $\begin{array}{c}1.52 \\
\pm 1.08\end{array}$ \\
\hline$\Omega_{\text {aragonite }}$ & $\begin{array}{c}1.91 \\
\pm 0.11\end{array}$ & $\begin{array}{c}0.35 \\
\pm 0.01\end{array}$ & $\begin{array}{c}1.83 \\
\pm 0.19\end{array}$ & $\begin{array}{c}0.35 \\
\pm 0.01\end{array}$ & $\begin{array}{c}1.69 \\
\pm 0.01\end{array}$ & $\begin{array}{c}0.36 \\
\pm 0.00\end{array}$ & $\begin{array}{c}1.03 \\
\pm 0.76\end{array}$ & $\begin{array}{c}1.74 \\
\pm 0.00\end{array}$ & $\begin{array}{c}1.74 \\
\pm 0.01\end{array}$ & $\begin{array}{c}1.74 \\
\pm 0.01\end{array}$ & $\begin{array}{c}1.59 \\
\pm 0.01\end{array}$ & $\begin{array}{c}0.38 \\
\pm 0.05\end{array}$ & $\begin{array}{c}0.98 \\
\pm 0.70\end{array}$ \\
\hline DIC $\left(\mu \mathrm{mol} \mathrm{l} \mathrm{l}^{-1}\right)$ & $\begin{array}{l}1810 \\
\pm 53\end{array}$ & $\begin{array}{l}1940 \\
\pm 53\end{array}$ & $\begin{array}{l}1840 \\
\pm 22\end{array}$ & $\begin{array}{l}1975 \\
\pm 15\end{array}$ & $\begin{array}{l}1770 \\
\pm 15\end{array}$ & $\begin{array}{l}1940 \\
\pm 48\end{array}$ & $\begin{array}{r}1850 \\
\pm 104\end{array}$ & $\begin{array}{l}1900 \\
\pm 91\end{array}$ & $\begin{array}{l}1720 \\
\pm 44\end{array}$ & $\begin{array}{r}1810 \\
\pm 125\end{array}$ & $\begin{array}{l}1812 \\
\pm 14\end{array}$ & $\begin{array}{r}1890 \\
\pm 3.0\end{array}$ & $\begin{array}{l}1850 \\
\pm 56\end{array}$ \\
\hline $\mathrm{CO}_{3}{ }^{2-}\left(\mu \mathrm{mol} \mathrm{l}{ }^{-1}\right)$ & $\begin{array}{c}118 \\
\pm 7.0\end{array}$ & $\begin{array}{c}21.4 \\
\pm 0.50\end{array}$ & $\begin{array}{r}113 \\
\pm 12\end{array}$ & $\begin{array}{r}21.5 \\
\pm 0.57\end{array}$ & $\begin{array}{c}104 \\
\pm 0.50\end{array}$ & $\begin{array}{c}22.4 \\
\pm 0.18\end{array}$ & $\begin{array}{l}63.3 \\
\pm 58\end{array}$ & $\begin{array}{c}107 \\
\pm 0.14\end{array}$ & $\begin{array}{c}107 \\
\pm 0.58\end{array}$ & $\begin{array}{c}107 \\
\pm 0.10\end{array}$ & $\begin{array}{c}97.9 \\
\pm 0.79\end{array}$ & $\begin{array}{r}23.3 \\
\pm 3.1\end{array}$ & $\begin{array}{l}60.6 \\
\pm 53\end{array}$ \\
\hline TA $\left(\mu \mathrm{mol} \mathrm{l}{ }^{-1}\right)$ & $\begin{array}{l}1970 \\
\pm 62\end{array}$ & $\begin{array}{l}1870 \\
\pm 44\end{array}$ & $\begin{array}{l}1990 \\
\pm 39\end{array}$ & $\begin{array}{l}1900 \\
\pm 9.6\end{array}$ & $\begin{array}{l}1910 \\
\pm 17\end{array}$ & $\begin{array}{l}1870 \\
\pm 44\end{array}$ & $\begin{array}{l}1890 \\
\pm 23\end{array}$ & $\begin{array}{l}2040 \\
\pm 87\end{array}$ & $\begin{array}{l}1870 \\
\pm 41\end{array}$ & $\begin{array}{r}1960 \\
\pm 119\end{array}$ & $\begin{array}{l}1940 \\
\pm 12\end{array}$ & $\begin{array}{l}1840 \\
\pm 13\end{array}$ & $\begin{array}{l}1890 \\
\pm 74\end{array}$ \\
\hline Salinity & $\begin{array}{r}29.3 \\
\pm 1.3\end{array}$ & $\begin{array}{r}29.3 \\
\pm 1.3\end{array}$ & $\begin{array}{r}29.3 \\
\pm 1.3\end{array}$ & $\begin{array}{r}29.3 \\
\pm 1.3\end{array}$ & $\begin{array}{r}29.3 \\
\pm 1.3\end{array}$ & $\begin{array}{r}29.3 \\
\pm 1.3\end{array}$ & $\begin{array}{r}29.3 \\
\pm 1.3\end{array}$ & $\begin{array}{r}29.3 \\
\pm 1.3\end{array}$ & $\begin{array}{r}29.3 \\
\pm 1.3\end{array}$ & $\begin{array}{r}29.3 \\
\pm 1.3\end{array}$ & $\begin{array}{r}29.3 \\
\pm 1.3\end{array}$ & $\begin{array}{r}29.3 \\
\pm 1.3\end{array}$ & $\begin{array}{r}29.3 \\
\pm 1.3\end{array}$ \\
\hline $\begin{array}{l}\text { Temperature } \\
\left({ }^{\circ} \mathrm{C}\right)\end{array}$ & $\begin{array}{r}22.8 \\
\pm 0.2\end{array}$ & $\begin{array}{r}22.8 \\
\pm 0.2\end{array}$ & $\begin{array}{r}22.8 \\
\pm 0.2\end{array}$ & $\begin{array}{r}22.8 \\
\pm 0.2\end{array}$ & $\begin{array}{r}22.8 \\
\pm 0.2\end{array}$ & $\begin{array}{r}22.8 \\
\pm 0.2\end{array}$ & $\begin{array}{r}22.8 \\
\pm 0.2\end{array}$ & $\begin{array}{r}22.8 \\
\pm 0.2\end{array}$ & $\begin{array}{r}22.8 \\
\pm 0.2\end{array}$ & $\begin{array}{r}22.8 \\
\pm 0.2\end{array}$ & $\begin{array}{r}22.8 \\
\pm 0.2\end{array}$ & $\begin{array}{r}22.8 \\
\pm 0.2\end{array}$ & $\begin{array}{r}22.8 \\
\pm 0.2\end{array}$ \\
\hline
\end{tabular}

and $0.4 \pm 0.4 \mu \mathrm{m} \mathrm{d}^{-1}$ in low $\mathrm{pH}(\mathrm{p}=0.014)$, diurnal $\mathrm{pH}$ $(\mathrm{p}<0.001)$, low $\mathrm{pH}-\mathrm{DO}(\mathrm{p}<0.001)$, and diurnal $\mathrm{pH}-\mathrm{DO}(\mathrm{p}<0.001)$ conditions, respectively (Fig. 4B). Growth rates of $C$. virginica larvae exposed to chron-

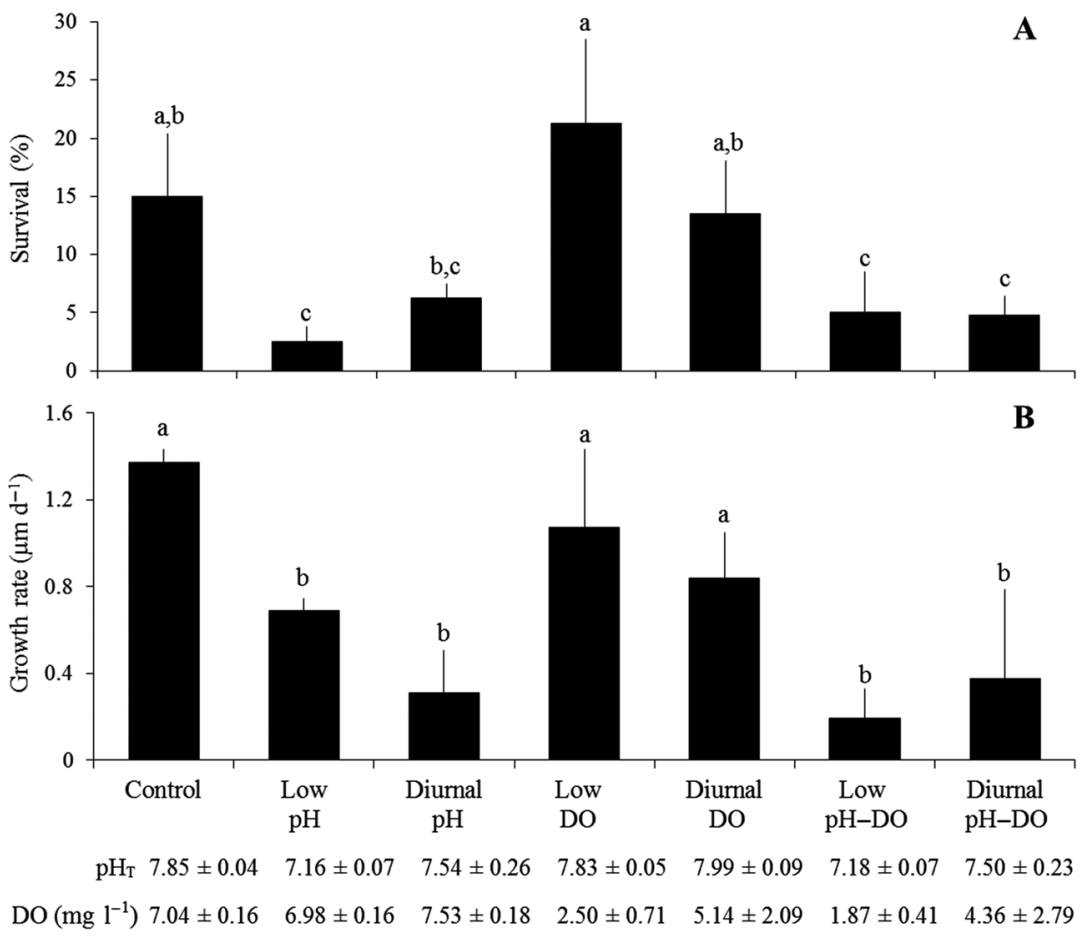

ically low or diurnal fluctuations of DO $(1 \pm 0.4$ and $1 \pm 0.2 \mu \mathrm{m} \mathrm{d}^{-1}$, respectively) did not differ from the control treatment. The interactive effect of $\mathrm{pH}$ and DO on C. virginica growth rates was most obvious in the diurnal treatments, where exposure to diurnally low $\mathrm{pH}$ and DO yielded growth rates higher than would have been predicted by the individual treatments. Metamorphic state was not quantified for $C$. virginica larvae.

\section{DISCUSSION}

Ocean acidification and hypoxia are expected to worsen as a result of anthropogenic activity (Diaz \&

Fig. 4. (A) Survival and (B) growth of Crassostrea virginica larvae. Error bars represent $\mathrm{SD}$ of the mean $(\mathrm{n}=4)$. Mean $\mathrm{pH}$ and DO values during the experiment for each treatment are shown, whereas detailed experimental conditions appear in Table 3. Letters above bars denote significant groupings of treatments as indicated via post hoc Tukey's HSD tests 
Rosenberg 2008, Breitburg et al. 2009, Doney et al. 2009). Both processes are known to negatively impact a multitude of marine species (e.g. Diaz \& Rosenberg 2008, Doney et al. 2009). Biotic controls of $\mathrm{pH}$ and DO in highly productive, coastal ecosystems complicate predictions of how ocean acidification and hypoxia will manifest in such environments in the future (Miller et al. 2009, Borges \& Gypens 2010, Duarte et al. 2013). To date, nearly all studies of bivalves have reported the independent effects of high $\mathrm{CO}_{2}$ or hypoxia, with only a few investigating effects of simultaneous exposure. No prior study has investigated the diurnal compared to continuous, as well as independent and combined effects of hypoxia and ocean acidification on bivalves. This study found that continuously low $\mathrm{pH}$ and low DO negatively impacted survival, growth, and development of larval bivalves, and the concurrent continuous exposure had both additively negative and interactive effects. Additionally, diurnal exposure to these conditions rarely improved the performance of larvae, despite significantly higher mean $\mathrm{pH}$ and DO levels. Collectively, these findings have important implications for understanding the effects of estuarine acidification and hypoxia on marine bivalves.

Continuously acidified conditions reduced survival of larvae of all 3 bivalve species more than low oxygen conditions. Acidification also slowed the growth of larval Argopecten irradians and Crassostrea virginica, and the development of A. irradians. Of the 3 species in this study, Mercenaria mercenaria was the most resistant to high $\mathrm{CO}_{2}$ as its growth was unaffected by $\mathrm{pH}$, a finding consistent with past studies (Talmage \& Gobler 2010). Shell formation and development of bivalve larvae is energetically costly and requires an even larger energy input when elevated $\mathrm{CO}_{2}$ creates unfavorable conditions for the precipitation of calcium carbonate (Palmer 1992, Pörtner 2008, Waldbusser et al. 2013). Waldbusser et al. (2013) reported that C. gigas larvae precipitated $90 \%$ of their body weight in calcium carbonate within the first $48 \mathrm{~h}$ of development, and some bivalve larvae precipitate a form of calcium carbonate that is less stable and more soluble than aragonite and calcite, known as amorphous calcium carbonate (Weiss et al. 2002). The high energetic cost of shell formation under elevated $\mathrm{CO}_{2}$ conditions may result in energy reallocation away from growth, resulting in smaller larvae with reduced lipid contents (Gobler \& Talmage 2013, Waldbusser et al. 2013). Beyond promoting unfavorable conditions for the biomineralization of calcium carbonate, hypercapnia can create other physiological problems for marine invertebrates. Dis- turbances in acid-base regulation, protein synthesis, and metabolism occur as a result of high $\mathrm{CO}_{2}$ exposure (Pörtner et al. 2005, Sokolova 2013, Waldbusser et al. 2015), and could further disrupt development and growth or induce mortality.

Coastal eutrophication is expected to worsen as a result of anthropogenic nutrient loading leading to increased size, duration, and severity of hypoxic regions (Diaz \& Rosenberg 2008, Rabalais et al. 2009). The results of this study show that continuously hypoxic conditions negatively affected survival, growth, and development of $A$. irradians larvae and slowed the development of larval $M$. mercenaria, demonstrating that while bivalves are some of the more hypoxia-tolerant marine organisms (Diaz \& Rosenberg 1995), early life stages are still susceptible to the deleterious effects of low oxygen conditions. Slowed growth has been observed in multiple species of early-life-stage bivalves exposed to hypoxia (Wang \& Widdows 1991), including A. irradians (Gobler et al. 2014). These negative effects may be a result of tradeoffs encumbered by physiological adaptations employed to survive hypoxia. Reducing oxygen demand via metabolic depression and switching from aerobic to anaerobic metabolism are typical responses to low oxygen availability for many marine invertebrates (Grieshaber et al. 1994, Guppy \& Withers 1999, Hochachka \& Lutz 2001). Although reduced metabolism and more energetically costly anaerobic metabolic pathways enable an organism to survive hypoxia or anoxia, growth may be inhibited as a result ( $\mathrm{Wu}$ 2002). Low DO concentrations used in these experiments $\left(\sim 2 \mathrm{mg} \mathrm{l}^{-1}\right)$, while considered hypoxic (Diaz 2001, Rabalais et al. 2002), may still be tolerable to some species. For example, although A. irradians larvae suffered reductions in survival, growth, and development under low DO conditions, there was no effect of low DO on survival or growth of M. mercenaria and C. virginica larvae, thus indicating species-specific tolerance of these conditions. These differences may be a function of the more rapid rates of growth and respiration in A. irradians compared to M. mercenaria and C. virginica (Kennedy et al. 1996, Kraeuter \& Castagna 2001, Shumway \& Parsons 2006).

Concurrent exposure to acidified and hypoxic water usually yielded a more negative outcome for larvae than each factor alone. Low $\mathrm{pH}-\mathrm{DO}$ yielded reduced survival in all 3 bivalve species examined, inhibited growth in $A$. irradians and $C$. virginica larvae, and repressed development in A. irradians and $M$. mercenaria larvae. The interactive effects of simultaneously low $\mathrm{pH}$ and low DO on the survival of 
Table 4. Overview of the effects of pH and DO on the survival, growth, and metamorphosis of Argopecten irradians, Mercenaria mercenaria, and Crassostrea virginica larvae. X: treatment that yielded a significantly lower performance compared to the level achieved in the control; -: treatment that yielded a performance that did not differ from the control; $\uparrow$ : diurnal treatment that yielded a performance significantly better than the continuous treatment, but significantly lower than the control treatment. Diurnal exposure significantly improved performance in $25 \%$ of instances, but never fully ameliorated the significant negative effects of low $\mathrm{pH}$ and/or low DO on larvae, despite higher mean $\mathrm{pH}$ and DO levels

\begin{tabular}{|c|c|c|c|c|c|c|}
\hline & Low $\mathrm{pH}$ & Diurnal pH & Low DO & Diurnal DO & Low $\mathrm{pH}, \mathrm{DO}$ & Diurnal pH, DO \\
\hline \multicolumn{7}{|l|}{ A. irradians } \\
\hline Survival & $\mathrm{X}$ & $\mathrm{X}$ & $\mathrm{X}$ & $\mathrm{X}$ & $\mathrm{X}$ & $\mathrm{X}$ \\
\hline Growth rate & $\mathrm{X}$ & $\uparrow$ & $\mathrm{X}$ & $\mathrm{X}$ & $\mathrm{X}$ & $\mathrm{X}$ \\
\hline Metamorphosis & $\mathrm{X}$ & $\uparrow$ & $\mathrm{X}$ & $\uparrow$ & $\mathrm{X}$ & $\uparrow$ \\
\hline \multicolumn{7}{|l|}{ M. mercenaria } \\
\hline Survival & $\mathrm{X}$ & $\mathrm{X}$ & - & - & $\mathrm{X}$ & $\mathrm{X}$ \\
\hline Growth rate & - & - & - & - & - & - \\
\hline Metamorphosis & $\mathrm{X}$ & $\mathrm{X}$ & $\mathrm{X}$ & $\mathrm{X}$ & $\mathrm{X}$ & $\mathrm{X}$ \\
\hline \multicolumn{7}{|l|}{ C. virginica } \\
\hline Survival & $\mathrm{X}$ & $\uparrow$ & - & - & $\mathrm{X}$ & $\mathrm{X}$ \\
\hline Growth rate & $\mathrm{X}$ & $\mathrm{X}$ & - & - & $\mathrm{X}$ & $\mathrm{X}$ \\
\hline
\end{tabular}

A. irradians and M. mercenaria larvae, development of M. mercenaria larvae, and growth of $C$. virginica larvae evidenced the complex physiological effects of these stressors on these bivalves. Interactions among multiple stressors may arise when the physiological pathways that the stressors act upon are not entirely independent. For example, exposure to low oxygen could reduce an organism's acid-base regulatory mechanisms, making them more susceptible to acidified conditions (Pörtner 2008). While the combined $\mathrm{pH}-\mathrm{DO}$ treatments usually yielded an outcome more severe than the individual treatments, the antagonistic effects observed during some experiments indicated that the combined effects were less severe than would have been predicted by the individual variables. This outcome suggests that some of the negative effects of $\mathrm{pH}$ and DO emanated from action on similar, rather than independent, physiological pathways. For example, if low $\mathrm{pH}$ only affected calcification, and low DO only affected aerobic metabolism, and these pathways were wholly independent of each other, then the combination of low pH-DO would have been strictly additive. Instead, the interactive effects observed suggest there is some level of overlap in the physiological impacts of these stressors, a hypothesis supported by prior studies. Acidification has been shown to reduce the lipid content and RNA:DNA ratios of bivalve larvae (Gobler \& Talmage 2013), suggesting a more universal, cascading physiological impact of low $\mathrm{pH}$ beyond simply inhibiting calcification. Given that low DO is also known to have large, overarching effects on bivalve physiology and metabolism (Diaz \& Rosenberg 1995, Vaquer-Sunyer \& Duarte 2008,
Levin et al. 2009), it seems likely that some of the physiological impacts of low DO and low $\mathrm{pH}$ overlap, accounting for the interactive effects on some traits of the bivalves studied here. Regardless, the compounded effects of hypoxia and acidification in a changing climate will ultimately favor bivalve species whose early life stages have the ability to adapt and maintain performance under shifting conditions (Pörtner \& Farrell 2008).

Diurnal fluctuations in $\mathrm{pH}$ and $\mathrm{DO}$ driven by ecosystem metabolism have commonly been observed in shallow estuaries (Ringwood \& Keppler 2002, Yates et al. 2007, Baumann et al. 2015), and climate change may make these fluctuations more extreme in the future (Rabalais et al. 2002, Miller et al. 2009, Feely et al. 2010). The extent to which the daily excursions to near normal levels of $\mathrm{pH}$ and DO may provide a temporal refuge for animals suffering from potential negative effects of these conditions is unknown at present. Given that diurnal exposure treatments during this study provided mean $\mathrm{pH}$ and DO levels that were significantly less severe than the chronic exposures, it would be expected that performance within diurnal exposure treatments would be improved relative to chronic exposures. Indeed, in $25 \%$ of possible outcomes (Table 4 ), the significant reductions in performance experienced by exposure to low $\mathrm{pH}$ and/or low DO were ameliorated by diurnal exposure. For example, the negative effects of low $\mathrm{pH}$ and low DO on the development of A. irradians larvae were significantly lessened when the exposure was ephemeral on diurnal timescales. Diurnal acidified conditions lessened the negative effects of low $\mathrm{pH}$ on survival of $C$. virginica larvae and on the growth of 
A. irradians. In all of these cases, however, the larval performance in these diurnal treatments was still significantly poorer than in the control, indicating that the diurnal exposure lessened, but did not eliminate, negative effects.

A substantially more common occurrence (75\% of outcomes; Table 4) were cases when chronically low DO and/or pH significantly inhibited the larval performance and outcomes were not improved by diurnal exposure, despite substantially and significantly higher mean $\mathrm{pH}$ and DO levels. This was the case for A. irradians survival when exposed to diurnal $\mathrm{pH}$, diurnal DO, and diurnal $\mathrm{pH}$ and $\mathrm{DO}$; for A. irradians growth when exposed to diurnal DO and diurnal $\mathrm{pH}$ and DO; for $M$. mercenaria survival when exposed to diurnal $\mathrm{pH}$ and diurnal $\mathrm{pH}$ and $\mathrm{DO}$; for $M$. mercenaria growth when exposed to diurnal $\mathrm{pH}$, diurnal DO, and diurnal $\mathrm{pH}$ and $\mathrm{DO}$; for $M$. mercenaria metamorphosis when exposed to diurnal $\mathrm{pH}$, diurnal $\mathrm{DO}$, and diurnal $\mathrm{pH}$ and $\mathrm{DO}$; for $C$. virginica survival when exposed to diurnal $\mathrm{pH}$ and $\mathrm{DO}$; and for C. virginica growth when exposed to diurnal $\mathrm{pH}$ and diurnal $\mathrm{pH}$ and DO. This outcome suggests that diurnally changing $\mathrm{pH}$ and DO may not allow enough time for bivalves to acclimate to the fluctuating conditions present during experiments. This may be particularly important in the case of diurnal DO changes, as it may cause bivalves to switch between aerobic and anaerobic metabolism (Grieshaber et al. 1994, Guppy \& Withers 1999, Hochachka \& Lutz 2001), an endeavor that may prove energetically costly. It is also possible that $12 \mathrm{~h}$ of exposure to suboptimal $\mathrm{pH}$ and/or DO conditions is enough to have detrimental physiological impacts. Nearly all studies of ocean acidification and hypoxia to date have exposed organisms to continuously high $\mathrm{CO}_{2}$ and/or low DO in laboratory settings (e.g. Waldbusser et al. 2013, Gobler et al. 2014), conditions that do not represent the manner in which some bivalves are exposed to acidification and hypoxia in many of the shallow estuaries they inhabit. However, our findings suggest that in most cases, diurnal exposure to low $\mathrm{pH}$ and low DO does not provide a refuge from the negative physiological impacts of these conditions.

The results of this study provide evidence of the differential negative impacts of chronic and diurnal fluctuations in low $\mathrm{pH}$ and DO on bivalves, as well as the interactions between these stressors. While growth rates of larvae within control treatments were within the ranges previously published (e.g. Gallager \& Mann 1986, Cahalan, et al. 1989, Helm et al. 2004), slower growth rates and delayed development leading to smaller organisms under acidified and/or hypoxic conditions may ultimately yield even higher rates of mortality in an ecosystem setting due to enhanced predation pressure (André \& Rosenberg 1991, Tamburri \& Zimmer-Faust 1996, Gosselin \& Qian 1997). In addition, longer-term effects of acidification and hypoxia on individuals surviving early life stage exposure may have negative 'carry over' or 'legacy' effects on subsequent life stages (Hettinger et al. 2012, Gobler \& Talmage 2013). All of these negative impacts could ultimately impact shellfish industries, as well as the many ecosystems services that bivalves provide (Officer et al. 1982, Petersen et al. 2015, Sebastiano et al. 2015).

Further research is needed to understand not only the long-term effects of acidification and hypoxia on shellfish, but also how anthropogenically driven acidification and hypoxia will manifest in coastal ecosystems. In the coming decades, progressive loading of anthropogenic $\mathrm{CO}_{2}$ from the atmosphere will lower ocean pH (Doney et al. 2009), while the excessive loading of nutrients in coastal ecosystems may decrease DO (Diaz 2001) and synergistically lower pH levels (Sunda \& Cai 2012). Alternatively, mitigation of nutrient loads could improve DO levels, while $\mathrm{pH}$ levels may be unchanged due to continued atmospheric loading. It has been proposed that anthropogenically driven acidification will depress the baseline, normal $\mathrm{pH}$ conditions in coastal ecosystems that go through diurnal changes (Miller et al. 2009, Feely et al. 2010), suggesting that even without changing nutrient loading, there may be some decoupling of $\mathrm{DO}$ and $\mathrm{pH}$ fluctuations in the future.

The diurnal ranges of $\mathrm{pH}$ and $\mathrm{DO}$ in estuaries depend upon many factors and differ among each coastal system. For example, Yates et al. (2007) reported diurnal changes in $\mathrm{pH}$ of 0.3 units and DO concentration of $\sim 2.0 \mathrm{mg}^{-1}$ in Tampa Bay, FL, USA, while Ringwood \& Keppler (2002) reported changes in $\mathrm{pH}$ on a diurnal cycle of 0.3 to 0.7 units and diurnal DO changes of 3 to $4 \mathrm{mg} \mathrm{l}^{-1}$ in Charleston Harbor, SC, USA. Baumann et al. (2015) demonstrated that the range of diurnal changes in $\mathrm{pH}$ and $\mathrm{DO}$ are seasonally dependent in temperate estuaries, being minimal in winter and maximal in late summer, with daily $\mathrm{pH}$ and DO changes of 0.7 units and $6.5 \mathrm{mg} \mathrm{l}^{-1}$, respectively, changes reflective of the conditions applied during the experiments presented in this study. There are many important considerations when comparing the experimental diurnal changes in this study to those that occur in estuaries. While the rate of $\mathrm{pH}$ changes during our experiments was somewhat gradual and thus consistent with ecosystem observations (e.g. Ringwood \& Keppler 2002, Bau- 
mann et al. 2015), DO changes were more rapid, occurring in $<1 \mathrm{~h}$ (Fig. 1), possibly decreasing the ability of larvae to adapt. Furthermore, while the experiments presented here returned larvae to ideal DO conditions for nearly $12 \mathrm{~h}$ each day, in some estuaries, the period of ideal conditions during summer can be significantly shorter (Baumann et al. 2015) or, in some cases, ideal conditions may never be achieved. This partly parallels our $\mathrm{pH}$ conditions, which were maximal for a shorter period of time than DO conditions (Fig. 1). This was also shown by Ringwood \& Keppler (2002), who demonstrated that $\mathrm{pH}$ varied by 0.3 to 0.7 units daily within South Carolina estuaries, and that the daily $\mathrm{pH}$ maximum never exceeded 7.6 on the NBS scale. Regardless, given that providing larvae with ideal DO conditions for nearly $12 \mathrm{~h}$ each day, and ideal $\mathrm{pH}$ for several hours each day, did not provide a refuge, it is possible the actual negative effects of $\mathrm{DO}$ and/or $\mathrm{pH}$ on early-lifestage bivalves may be due to a duration of time below a critical threshold value. Ultimately, there are an infinite number of potential ecosystem and experimental combinations regarding exposure duration and severity of acidification and hypoxia, leaving much to be learned regarding the effects of diurnal $\mathrm{pH}$ and DO exposure on marine life. The levels and duration of low $\mathrm{pH}$ and low DO used in experiments presented here were, in many ways, consistent with prior ecosystem observations (Ringwood \& Keppler 2002, Baumann et al. 2015), and were detrimental to the growth and survival of North Atlantic bivalve larvae.

Although this study focused on diurnal acidification and hypoxia, it is important to note that not all shallow coastal systems experience biotic-driven diurnal variations in $\mathrm{pH}$ and $\mathrm{DO}$. Other factors, such as acidic and low salinity riverine discharge, can intermittently influence $\mathrm{pH}$ only (Salisbury et al. 2008), and tidal processes in estuaries can alter $\mathrm{pH}$ and DO on cycles that may act synergistically or antagonistically with diurnal cycles (Baumann et al. 2015). Additionally, in deeper stratified water, hypoxia and acidification are not alleviated via photosynthetic activity, and can persist for long periods of time (weeks to months; Rabalais et al. 2002, Diaz \& Rosenberg 2008, Wallace et al. 2014).

In conclusion, diurnal exposure of larval bivalves indigenous to the Northwest Atlantic Ocean to low $\mathrm{pH}$ and low DO infrequently alleviated the effects of low $\mathrm{DO}$ and low $\mathrm{pH}$, despite the higher mean $\mathrm{pH}$ and DO levels in those treatments. Regardless of the manner in which larval bivalves are exposed to acidic and hypoxic conditions, they experienced deleterious effects. Given that shellfish are ecologically and economically important marine organisms, implementing managerial criteria that will mitigate anthropogenic acidification and hypoxia seems warranted.

Acknowledgements. This work was supported by NOAA's Ocean Acidification Program through award \#NA12NOS 4780148 from the National Centers for Coastal Ocean Science, New York Sea Grant Award R-FBM-38, the Laurie Landeau Foundation, the Simons Foundation, and the Chicago Community Trust. The authors have no significant competing financial, professional, or personal interests that might have influenced the materials presented in this manuscript. The authors gratefully acknowledge the assistance of Andrew Griffith, Robert McCabe, Heidi Mittelsdorf, Elizabeth Depasquale, Mark Lusty, and Ryan Wallace. We thank Dr. Jeffery Levinton and Dr. Glenn Lopez for helpful feedback on this manuscript and study, as well as the editor and 2 anonymous reviewers for their useful comments.

\section{LITERATURE CITED}

André C, Rosenberg R (1991) Adult-larval interactions in the suspension-feeding bivalves Cerastoderma edule and Mya arenaria. Mar Ecol Prog Ser 71:227-234

Baumann H, Wallace RB, Tagliaferri T, Gobler CJ (2015) Large natural $\mathrm{pH}, \mathrm{CO}_{2}$ and $\mathrm{O}_{2}$ fluctuations in a temperate tidal salt marsh on diel, seasonal, and interannual time scales. Estuar Coasts 38:220-231

Borges AV, Gypens N (2010) Carbonate chemistry in the coastal zone responds more strongly to eutrophication than ocean acidification. Limnol Oceanogr 55:346-353

Breitburg DL, Hondorp DW, Davias LA, Diaz RJ (2009) Hypoxia, nitrogen, and fisheries: integrating effects across local and global landscapes. Annu Rev Mar Sci 1: 329-349

Cahalan JA, Siddall SE, Luckenbach MW (1989) Effects of flow velocity, food concentration and particle flux on growth rates of juvenile bay scallops Argopecten irradians. J Exp Mar Biol Ecol 129:45-60

> Cai WJ, Wang Y (1998) The chemistry, fluxes, and sources of carbon dioxide in the estuarine waters of the Satilla and Altamaha Rivers, Georgia. Limnol Oceanogr 43:657-668

$>$ Cai WJ, Hu X, Huang WJ, Murrell MC and others (2011) Acidification of subsurface coastal waters enhanced by eutrophication. Nat Geosci 4:766-770

Caldeira K, Wickett ME (2003) Oceanography: anthropogenic carbon and ocean $\mathrm{pH}$. Nature 425:365

Carriker MR (2001) Embryogenesis and organogenesis of veligers and early juveniles. In: Kraeuter JN, Castagna $M$ (eds) Biology of the hard clam. Elsevier Science, New York, NY, p 77-115

Cragg SM (2006) Development, physiology, behavior, and ecology of scallop larvae. In: Shumway SE, Parsons GJ (eds) Scallops: biology, ecology, and aquaculture. Elsevier, New York, NY, p 45-122

> Diaz RJ (2001) Overview of hypoxia around the world. J Environ Qual 30:275-281

Diaz RJ, Rosenberg R (1995) Marine benthic hypoxia: a review of its ecological effects and the behavioral responses of benthic macrofauna. Oceanogr Mar Biol 
Annu Rev 33:245-303

Diaz RJ, Rosenberg R (2008) Spreading dead zones and consequences for marine ecosystems. Science 321:926-929

$>$ Dickson AG (1993) pH buffers for sea-water media based on the total hydrogen-ion concentration scale. Deep-Sea Res I 40:107-118

> Doney SC, Fabry VJ, Feely RA, Kleypas JA (2009) Ocean acidification: the other $\mathrm{CO}_{2}$ problem. Annu Rev Mar Sci 1:169-192

> Duarte CM, Hendriks IE, Moore TS, Olsen YS and others (2013) Is ocean acidification an open-ocean syndrome? Understanding anthropogenic impacts on seawater $\mathrm{pH}$. Estuar Coasts 36:221-236

Feely RA, Alin SR, Newton J, Sabine CL and others (2010) The combined effects of ocean acidification, mixing, and respiration on $\mathrm{pH}$ and carbonate saturation in an urbanized estuary. Estuar Coast Shelf Sci 88:442-449

- Gazeau F, Parker LM, Comeau S, Gattuso JP and others (2013) Impacts of ocean acidification on marine shelled molluscs. Mar Biol 160:2207-2245

> Gobler CJ, Talmage SC (2013) Short-and long-term consequences of larval stage exposure to constantly and ephemerally elevated carbon dioxide for marine bivalve populations. Biogeosciences 10:2241-2253

Gobler CJ, DePasquale EL, Griffith AW, Baumann H (2014) Hypoxia and acidification have additive and synergistic negative effects on the growth, survival, and metamorphosis of early life stage bivalves. PLOS ONE 9:e83648

Gosselin LA, Qian PY (1997) Juvenile mortality in benthic marine invertebrates. Mar Ecol Prog Ser 146:265-282

Grieshaber MK, Hardewig I, Kreutzer U, Pörtner HO (1994) Physiological and metabolic responses to hypoxia in invertebrates. Rev Physiol Biochem Pharmacol 125:43-147

Guppy M, Withers P (1999) Metabolic depression in animals: physiological perspectives and biochemical generalizations. Biol Rev Camb Philos Soc 74:1-40

Helm MM, Bourne N, Lovatelli A (2004) Hatchery culture of bivalves: a practical manual. Food and Agriculture Organization of the United Nations, Rome

> Hettinger A, Sanford E, Hill TM, Russell AD (2012) Persistent carry-over effects of planktonic exposure to ocean acidification in the Olympia oyster. Ecology 93:2758-2768

> Hochachka PW, Lutz PL (2001) Mechanism, origin, and evolution of anoxia tolerance in animals. Comp Biochem Physiol B 130:435-459

Kennedy VS, Newell RIE, Eble AF (1996) The eastern oyster: Crassostrea virginica. Maryland Sea Grant College, College Park, MD

Keppel AG, Breitburg DL, Wikfors GH, Burrell RB, Clark VM (2015) Effects of co-varying diel-cycling hypoxia and $\mathrm{pH}$ on disease susceptibility in the eastern oyster Crassostrea virginica. Mar Ecol Prog Ser 538:169-183

Kim T, Barry J, Micheli F (2013) The effects of intermittent exposure to low-pH and low-oxygen conditions on survival and growth of juvenile red abalone. Biogeosciences 10:7255-7262

Kraeuter JN, Castagna M (2001) Biology of the hard clam. Elsevier Science, New York, NY.

> Levin LA, Ekau W, Gooday AJ, Jorissen F and others (2009) Effects of natural and human-induced hypoxia on coastal benthos. Biogeosciences 6:2063-2098

Melzner F, Thomsen J, Koeve W, Oschlies A and others (2013) Future ocean acidification will be amplified by hypoxia in coastal habitats. Mar Biol 160:1875-1888

Michaelidis B, Ouzounis C, Paleras A, Pörtner HO (2005)
Effects of long-term moderate hypercapnia on acid-base balance and growth rate in marine mussels Mytilus galloprovincialis. Mar Ecol Prog Ser 293:109-118

Miller AW, Reynolds AC, Sobrino C, Riedel GF (2009) Shellfish face uncertain future in high $\mathrm{CO}_{2}$ world: influence of acidification on oyster larvae calcification and growth in estuaries. PLOS ONE 4:e5661

Millero FJ (2010) Carbonate constants for estuarine waters. Mar Freshw Res 61:139-142

Officer CB, Smayda TJ, Mann R (1982) Benthic filter feeding: a natural eutrophication control. Mar Ecol Prog Ser 9:203-210

> Palmer AR (1992) Calcification in marine mollusks: how costly is it? Proc Natl Acad Sci USA 89:1379-1382

> Petersen JK, Saurel C, Nielsen P, Timmermann K (2015) The use of shellfish for eutrophication control. Aquacult Int 24:857-878

> Pörtner HO (2008) Ecosystem effects of ocean acidification in times of ocean warming: a physiologist's view. Mar Ecol Prog Ser 373:203-217

Pörtner HO, Farrell AP (2008) Physiology and climate change. Science 322:690-692

> Pörtner HO, Langenbuch M, Michaelidis B (2005) Synergistic effects of temperature extremes, hypoxia, and increases in $\mathrm{CO}_{2}$ on marine animals: from Earth history to global change. J Geophys Res, C, Oceans 110:C09S10

$>$ Rabalais NN, Turner RE, Wiseman WJ (2002) Gulf of Mexico hypoxia, a.k.a. "The dead zone". Annu Rev Ecol Syst 33: 235-263

> Rabalais NN, Turner RE, Díaz RJ, Justíc D (2009) Global change and eutrophication of coastal waters. ICES J Mar Sci 66:1528-1537

> Ringwood AH, Keppler CJ (2002) Water quality variation and clam growth: is $\mathrm{pH}$ really a non-issue in estuaries? Estuaries 25:901-907

Sabine CL, Feely RA, Gruber N, Key RM and others (2004) The oceanic sink for anthropogenic $\mathrm{CO}_{2}$. Science 305: 367-371

Salisbury J, Green MA, Hunt C, Campbell J (2008) Coastal acidification by rivers: a threat to shellfish? Eos Trans AGU 89:513-528

Sebastiano D, Levinton JS, Doall M, Kamath S (2015) Using a shellfish harvest strategy to extract high nitrogen inputs in urban and suburban coastal bays: practical and economic implications. J Shellfish Res 34:573-583

Shumway SE, Parsons GJ (2006) Scallops: biology, ecology, and aquaculture. Elsevier, Boston, MA

Sokolova IM (2013) Energy-limited tolerance to stress as a conceptual framework to integrate the effects of multiple stressors. Integr Comp Biol 53:597-608

> Steckbauer A, Ramajo L, Hendriks IE, Fernandez M and others (2015) Synergistic effects of hypoxia and increasing $\mathrm{CO}_{2}$ on benthic invertebrates of the central Chilean coast. Front Mar Sci 2:49

> Sunda WG, Cai WJ (2012) Eutrophication induced $\mathrm{CO}_{2}$ acidification of subsurface coastal waters: interactive effects of temperature, salinity, and atmospheric $p \mathrm{CO}_{2}$. Environ Sci Technol 46:10651-10659

Talmage SC, Gobler CJ (2009) The effects of elevated carbon dioxide concentrations on the metamorphosis, size, and survival of larval hard clams (Mercenaria mercenaria), bay scallops (Argopecten irradians), and eastern oysters (Crassostrea virginica). Limnol Oceanogr 54: 2072-2080

Talmage SC, Gobler CJ (2010) Effects of past, present, and 
future ocean carbon dioxide concentrations on the growth and survival of larval shellfish. Proc Natl Acad Sci USA 107:17246-17251

Tamburri MN, Zimmer-Faust RK (1996) Suspension feeding: basic mechanisms controlling recognition and ingestion of larvae. Limnol Oceanogr 41:1188-1197

> Vaquer-Sunyer R, Duarte CM (2008) Thresholds of hypoxia for marine biodiversity. Proc Natl Acad Sci USA 105: 15452-15457

> Waldbusser GG, Salisbury JE (2014) Ocean acidification in the coastal zone from an organism's perspective: multiple system parameters, frequency domains, and habitats. Annu Rev Mar Sci 6:221-247

Waldbusser GG, Voigt EP, Bergschneider H, Green MA, Newell RIE (2011) Biocalcification in the eastern oyster (Crassostrea virginica) in relation to long-term trends in Chesapeake Bay pH. Estuar Coasts 34:221-231

Waldbusser GG, Brunner EL, Haley BA, Hales B and others (2013) A developmental and energetic basis linking larval oyster shell formation to acidification sensitivity. Geophys Res Lett 40:2171-2176

Waldbusser GG, Hales B, Langdon CJ, Haley BA and others (2015) Ocean acidification has multiple modes of action on bivalve larvae. PLOS ONE 10:e0128376

> Wallace RB, Baumann H, Grear JS, Aller RC, Gobler CJ (2014) Coastal ocean acidification: the other eutrophication problem. Estuar Coast Shelf Sci 148:1-13

Wang WX, Widdows J (1991) Physiological responses of mussel larvae Mytilus edulis to environmental hypoxia and anoxia. Mar Ecol Prog Ser 70:223-236

Editorial responsibility: Myron Peck,

Hamburg, Germany
Wang WX, Widdows J (1993) Calorimetric studies on the energy-metabolism of an infaunal bivalve, Abra tenius, under normoxia, hypoxia and anoxia. Mar Biol 116: 73-79

Wang ZHA, Wanninkhof R, Cai WJ, Byrne RH and others (2013) The marine inorganic carbon system along the Gulf of Mexico and Atlantic coasts of the United States: insights from a transregional coastal carbon study. Limnol Oceanogr 58:325-342

Weiss IM, Tuross N, Addadi L, Weiner S (2002) Mollusc larval shell formation: amorphous calcium carbonate is a precursor phase for aragonite. J Exp Zool 293:478-491

White MM, McCorkle DC, Mullineaux LS, Cohen AL (2013) Early exposure of bay scallops (Argopecten irradians) to high $\mathrm{CO}_{2}$ causes a decrease in larval shell growth. PLOS ONE 8:e61065

- Wootton JT, Pfister CA, Forester JD (2008) Dynamic patterns and ecological impacts of declining ocean $\mathrm{pH}$ in a highresolution multi-year dataset. Proc Natl Acad Sci USA 105:18848-18853

Wu RSS (2002) Hypoxia: from molecular responses to ecosystem responses. Mar Pollut Bull 45:35-45

Yates KK, Dufore C, Smiley N, Jackson C, Halley RB (2007) Diurnal variation of oxygen and carbonate system parameters in Tampa Bay and Florida Bay. Mar Chem 104:110-124

> Zhang J, Gilbert D, Gooday AJ, Levin L and others (2010) Natural and human-induced hypoxia and consequences for coastal areas: synthesis and future development. Biogeosciences 7:1443-1467

Submitted: February 29, 2016; Accepted: July 28, 2016 Proofs received from author(s): September 17, 2016 